\title{
L'appréciation de la beauté : revue de littérature
}

\author{
Jacques FAURE ${ }^{*}$, Yves BOLENDER ${ }^{2}$ \\ 110 place Lannes, 32021 Auch, France \\ 2 Faculté de Chirurgie Dentaire, 1 place de l'Hôpital, 67000 Strasbourg, France
}

\section{MOTS CLÉS :}

Esthétique faciale /

Morphologie faciale /

Jugement esthétique /

Imagerie

tridimensionnelle /

Diagnostic

tridimensionnel /

Attrait facial

\section{KEYWORDS:}

Facial esthetics /

Facial morphology /

Esthetic judgment /

Three dimensional

Imaging /

Three dimensional

diagnosis /

Facial attractiveness
RÉSUMÉ - Le jugement esthétique est bien sûr subjectif, mais ceci n'exclut pas une étude rigoureuse mettant en jeu des méthodes scientifiques. La base factuelle de la «science de l'esthétique » ne doit pas établir qu'une personne ou une image est « objectivement » belle, mais elle doit plutôt déterminer comment un groupe social représentatif ou des jurys ressentent cet « objet esthétique » comme beau ou laid. Le but de cet article est la revue des aspects définitionnels, théoriques et méthodologiques de la perception par un groupe social homogène et représentatif, du caractère attractif d'un visage ou d'un sourire. La première partie pose les principes de l'attirance faciale : la perception suppose un jury, un champ d'investigation, et un test susceptible de produire des données quantifiées; les déterminants généraux de la beauté sont ensuite passés en revue : la morphologie moyenne, le background culturel du juge, la numérologie, l'origine ethnique du juge. Les déterminants indirects qui peuvent intervenir sont la denture, l'architecture osseuse et l'enveloppe musculaire. Des facteurs perturbateurs altérant le jugement peuvent être mis en cause ; ce sont des facteurs périphériques comme la coiffure et la couleur des cheveux, la texture de la peau, les rides, le rouge à lèvres... ou des facteurs psycho-sociaux comme l'aura du sujet évalué, son intelligence, son comportement. La seconde partie traite de la méthodologie employée pour apprécier «l'attractivité » d'une face et la corréler avec telle ou telle morphologie. Classiquement ceci est réalisé en déterminant la préférence esthétique moyenne pour divers groupes d'images faciales au sein d'un jury qui a pour tache spécifique d'estimer leurs qualités esthétiques. L'échantillon à juger, la présentation des documents, le ou les jurys, la procédure de notation sont discutés; ils doivent être précisés avant de commencer la collecte des données. Une attention spécifique est enfin portée sur la démarche d'appréciation associée à telle ou telle morphologie, décrite par des méthodes morpho-métriques. L'objectif ultime est bien sûr la sélection des morphologies les plus attractives pour nos patients.

ABSTRACT - Beauty judgment: revue of the literature. Esthetic judgments are surely subjective, but as surely, that does not preclude them being studied objectively through rigorous scientific methods. The factual basis of a science of esthetics is not to settle whether some person or image is "objectively beautiful" but rather to determine whether some representative set or sets of individuals judge or experience him/her/it as beautiful or unattractive. The aim of this paper is to review the definitional, theoretical and methodological aspects pertaining to the perception of facial/dental attractiveness by a group of representative individuals. The first part lays down the basic principles of the perception of facial/dental attractiveness: the perception involves a jury, a field of investigation and a test providing quantitative data; the following general determinants of beauty perception are reviewed: the average morphology, the judge's cultural background, the numerology, the judge's ethnical origin. Indirect determinants are the dentition, the osseous architecture and the muscular envelope. Some disruptive factors might alter the judges' facial perception.

* Auteur pour correspondance : j.h.faure@free.fr 


\begin{abstract}
They might be qualified as either peripheral to the face or psycho-social factors. Peripheral factors include hair style and color, skin hue, wrinkles, lips color... Psychosocial factors cover the personality of the subject being evaluated, his/her intelligence or behavior. The second part deals specifically with the methodology used to determine facial attractiveness and to correlate this latter with a specific morphology. Typically such a study aims to determine average esthetic preferences for some set of visual displays among a particular jury, given a specific task to judge esthetic quality or qualities. The sample being studied, the displays, the jury or jurys, the rating procedure must all be specified prior to collecting data. A specific emphasis will be given to the rating process and the associated morphometrics, the ultimate goal being to discriminate morphologies judged as attractive among our patients.
\end{abstract}

\section{Introduction}

La notion de beau nous est assenée par les philosophes comme une évidence qui s'imposerait à tous, une vérité absolue auto-proclamée, une intuition divinatrice clairement lisible pour chacun de nous. La beauté est souvent rapprochée de la rationalité ou de notions morales. Certains rapprochent aussi l'impression esthétique d'une saisie sensitive intuitive et globale (tactile, visuelle, auditive). Mais si la beauté était une notion si évidente et consensuelle, comment expliquer la disparité des jugements, des choix esthétiques, vestimentaires, par exemple?

Lorsqu'on examine plus attentivement les définitions proposées, les choses deviennent beaucoup moins évidentes. Souvent, dans les dictionnaires, les définitions de l'esthétique et de la beauté se renvoient la balle l'une à l'autre!

Enfin, lorsqu'il s'agit non pas d'un paysage naturel, d'un objet usuel ou même d'un objet d'art, mais d'un sujet porteur d'une lourde charge symbolique, comme notre silhouette, notre visage ou notre sourire, l'appréciation de la beauté devient beaucoup plus complexe.

De plus, d'une manière générale, la conception de l'esthétique a considérablement évolué au début du $20^{e}$ siècle dans le sens d'une « socialisation ». L'émotion artistique conçue jusqu'au $19^{e}$ siècle comme une communion entre l'artiste et le spectateur ne concerne qu'une élite cultivée. Les héritiers du marxisme, puis l'école de Francfort vont prendre conscience de l'intrusion des masses dans le domaine de l'art, les premiers pour réécrire l'histoire matérialiste et le devenir de l'art, les seconds pour idéaliser l'art et le défendre contre la «massification». Les générations ultérieures vont analyser la vie artistique à travers les rapports œuvre d'art/société, puis se pencher sur l'étude scientifique «épidémiologique » des demandes et des besoins esthétiques de la Société à l'aide de tests et d'enquêtes.

Nous examinerons dans une première partie les fondements de l'esthétique sociale, qui repose sur un processus complexe et non simple et intuitif. Après discussion de la notion fondatrice de jugement, nous évoquerons le cadre d'étude, la morphologie faciale et elle seule, et les facteurs déterminants principaux, accessoires et perturbateurs.

Nous exposerons ensuite les moyens à mettre en œuvre afin d'émettre un jugement esthétique sur la morphologie faciale, avec deux grands volets :

1. Les modalités de jugement.

2. Les analyses de forme associées à l'objet étudié.

Le but terminal est bien sûr le rapprochement de la note esthétique et de la forme, dans un but diagnostique comme thérapeutique.

\section{Les bases de l'esthétique sociale}

\subsection{Le jugement}

\subsubsection{Sémantique : morphologie ou esthétique}

Une première précision sémantique apporte un éclaircissement au problème : la différence entre esthétique et morphologie. Comme pour beaucoup d'autres professions, les orthodontistes confondent les deux concepts. Avoir un grand nez est une constatation morphologique. Faire une chirurgie de diminution ou d'augmentation est encore une réalité morphologique. Cela se mesure avec un pied à coulisse.

Le patient est-il beau avec son grand nez? La modification mesurée du nez a-t-elle amélioré ou dégradé l'esthétique faciale du patient? Cela requiert un jugement. 
Un travail de recherche sur la modification quantifiée des positions labiales en fonction du déplacement thérapeutique des incisives est un travail de morphologie. Le résultat s'exprime par des millimètres. Si l'amélioration de la note esthétique est mesurée (test), alors c'est un travail sur l'esthétique.

La différence est nette: morphologie implique mesure/esthétique implique jugement.

Mais cette constatation faite, force est de réaliser que les deux choses sont liées surtout pour l'orthodontiste : un travail sur l'esthétique repose sur deux volets : une analyse de la forme et un jugement. La forme constitue pour l'orthodontiste le centre d'intérêt de son exercice, la base de l'esthétique sous son contrôle, le moyen d'amélioration thérapeutique, les autres éléments (couleur, pilosité, texture cutanée, accessoires cosmétiques, personnalité...) étant hors de son domaine.

De plus, la littérature scientifique orthodontique oublie de faire la différence, et on trouve souvent avec un titre contenant explicitement le mot « esthétique »:

- des considérations morphologiques générales, souvent intéressantes, mais sans référence suffisante à une préférence sociale exprimée [30,44],

- des analyses des remaniements morphologiques thérapeutiques sans référence à un jugement quelconque.

\subsubsection{Jugement}

\subsubsection{Jugement individuel}

Esthétique suppose jugement, jugement porté par un sujet sur un objet. L'objet étant donné, le jugement esthétique est un événement aléatoire qui dépend du sujet. La note qui concrétise ce jugement varie selon le juge, c'est une variable aléatoire.

Le jugement esthétique sera conditionné :

- d'une part, par l'objet, avec son contenu objectif, c'est à dire ses paramètres physiques (couleur et forme pour un objet plastique) et ses paramètres subjectifs que sont son contenu symbolique, les références socioculturelles qu'il évoque;

- d'autre part, par le sujet, avec sa lecture objective des paramètres physiques et son interprétation personnelle liée à son background culturel.

\subsubsection{Esthétique sociale}

Si le jugement esthétique individuel est un événement aléatoire, le jugement porté par un groupe social défini est une réalité scientifique. Lorsqu'un groupe social est bien défini et si son comportement est homogène, alors il est possible de préciser son comportement, c'est à dire de connaître son jugement moyen et plus largement la loi de probabilité de la variable aléatoire note ou score esthétique (moyenne et écart-type).

\subsubsection{Moyens d'appréciation: les tests}

Le seul moyen d'apprécier l'esthétique sociale est bien évidemment, et les psychologues le savent depuis longtemps, l'emploi de tests. Le test consulte un groupe de personnes pour définir le jugement du groupe social.

En effet, chacun est capable de porter un jugement personnel sur un objet, mais nul n'est détenteur d'un œil susceptible d'apprécier sans erreur l'opinion moyenne, l'opinion sociale; nul n'est doué $\mathrm{du}$ « regard social moyen ». Les modalités de ces tests constituent l'objet même de cet article.

\subsubsection{Spécificité du visage-objet}

Les tests pour l'appréciation de l'attrait d'un objet sont largement utilisés par les spécialistes du marketing. Mais le visage-objet est un objet très spécifique : c'est l'outil essentiel de la communication et de la séduction pour l'homme.

L'objet d'art est déjà empreint d'une charge symbolique forte, que n'a pas, bien sûr, un objet naturel (fleur, paysage...); a fortiori le visage supporte une charge symbolique encore plus importante; en outre, il est largement modifiable grâce aux cosmétiques, aux lentilles de couleurs, à la coiffure, au contrôle de la mimique, à la chirurgie plastique... Le sujet va modifier son aspect facial pour améliorer l'esthétique mais aussi pour renforcer le message symbolique.

Le jugement esthétique porté sur le visage sera donc bien plus complexe que pour un objet quelconque.

Il faudra éliminer les déterminants qui ne relèvent pas de notre spécialité (coiffure, couleur des yeux...), pour ne garder que les éléments morphologiques sous notre contrôle. Il faudra absolument gommer 
l'intervention de la personnalité du sujet jugé, son aura, sa séduction personnelle et ne pas confondre beauté du visage et séduction.

\subsection{Le champ}

Le champ ou le domaine de notre étude et de notre prise en compte de l'esthétique sont liés à nos possibilités d'intervention thérapeutique et à la fréquence de ces interventions. La hiérarchie est bien sûr :

\section{Esthétique dentaire.}

2. Esthétique bucco-dentaire ou du sourire.

3. Esthétique faciale.

4. Esthétique du visage et de l'ensemble céphalique.

5. Esthétique globale de l'individu in toto.

Les documents de travail pour une étude scientifique comme pour un examen clinique à visée thérapeutique devront permettre l'appréciation des trois premiers champs; mais les derniers domaines, même s'ils ne sont pas sous contrôle éventuel de la thérapeutique, doivent être pris en compte puisqu'ils sont susceptibles d'influencer l'évaluation des premiers champs; nous envisagerons cela plus loin, dans la partie « documents».

Remarquons que cette hiérarchie correspond à celle des champs au sens du langage des photographes. L'observation en champ resserré permet, écarteurs en place, d'observer la denture seule; le champ moyen correspond à la photographie sousnasale (bouche : lèvres et dents découvertes par le sourire, incluses; nez et menton, exclus); en élargissant le champ, on a successivement la face, puis le visage et enfin l'ensemble du personnage.

La notion de champ est très importante dans l'appréciation esthétique faciale, surtout pour l'appréciation d'un résultat thérapeutique. Un patient peut avoir en fin de traitement un sourire bucco-dentaire fort agréable avec une esthétique faciale disgracieuse, dans un cas en principe chirurgical traité par une thérapeutique de «camouflage», par exemple. Lappréciation du résultat global appelle un jugement « grand champ » et des jugements « champ moyen » et « champ resserré ». Mais ces jugements ne sont pas indépendants bien sûr : l'esthétique bucco-dentaire participe à l'impression de beauté du visage, et elle bénéficie elle-même du bonus apporté par un environnement facial harmonieux.
Deux questions se posent.

Quelles sont les corrélations existant entre note du sujet en pied, note du visage du sujet, de la face, du sourire bucco-dentaire, de la denture?

Quelle est la hiérarchie de la participation des différents éléments anatomiques dans l'esthétique globale?

Là encore peu d'auteurs comparent les jugements portés séparément sur l'ensemble du visage souriant, sur le sourire bucco-dentaire et sur la denture seule [50]. La corrélation entre les notes des sourires bucco-dentaires et celles des vues endo-buccales de face est étroite, tandis que celle de ces deux notes avec les notes de l'esthétique faciale globale est plus faible. Ceci souligne l'importance de la denture dans le sourire, et relativise l'importance des éléments bucco-dentaires ou strictement dentaires dans l'appréciation globale.

Certains auteurs ont utilisé des méthodes d'enquêtes peu convaincantes pour hiérarchiser les éléments anatomiques faciaux les plus importants (nez, yeux, menton, lèvres...).

Afin d'apprécier l'influence de facteurs autres que les facteurs morphologiques, Spyropoulos [157] emploie des photographies de patients sans déformations et avec déformations (warping) leur donnant le profil moyen de l'échantillon tout en conservant leurs caractéristiques propres sur le plan coiffure, texture cutanée... Elle utilise aussi trois profils « composites», c'est à dire ayant une ligne du profil conforme à la ligne moyenne et des attributs esthétiques (peau, couleur des yeux) conformes à la moyenne de l'échantillon (morphing), mais des coiffures optimales copiées sur un autre cas.

Elle montre ainsi que le fait de donner un profil standard aux photographies originales des patients apporte un gain d'environ un point (échelle de notation esthétique de 1 à 10), alors qu'une amélioration de la coiffure leur fait gagner plus de deux points!

\subsection{La dynamique}

Le visage humain est animé, et ses mimiques sont un élément important de sa beauté ; comment apprécier la beauté d'un visage si l'on ne voit pas le sourire du sujet? Lappréciation esthétique doit donc porter sur le visage statique, mais aussi en mouvement et surtout souriant.

La saisie d'une modification morphologique rapide pose le difficile problème de l'intégration du 
temps. L'examen d'un sourire en vue d'un jugement esthétique et d'une rééducation fonctionnelle par exemple va présenter deux difficultés :

- Lexigence d'un examen dynamique et de documents cinématographiques $[1,2,145,172,173]$.

- Le choix de points-repères cutanés et de paramètres spécifiques (vecteur: direction et vitesse de déplacement commissurale par exemple).

Les spécialistes de communication pour les malentendants savent prendre en compte ce type de problèmes; une équipe s'est intéressée à ce thème de recherche $[172,173]$.

N'oublions pas non plus de ne pas limiter notre attention au sourire. La création d'un sculpteur Frantz Xavier Messerschmidt, qui toute sa vie durant a étudié et décrit les différentes mimiques humaines (douleur, angoisse, mépris...), devrait nous inciter à nous pencher plus encore sur l'ensemble de la dynamique du visage. N'oublions pas que, dans la vie sociale, le visage en face de nous n'est pas une seconde au repos; donc une appréciation esthétique favorable suppose un système de mimiques globalement très agréable. Un grand psychologue, Paul Ekman, s'est penché sur l'analyse des mimiques en identifiant d'abord sept puis, plus finement, seize types différents et en associant à chaque type identifié le codage des muscles faciaux mis en œuvre, dans la région des yeux et dans la région buccale principalement $[45,46]$.

\subsection{Les facteurs}

Il est certain que l'appréciation de la beauté exige la compréhension des processus de préférence esthétique, avant d'entrer dans l'étude des méthodes de mesure.

Les facteurs déterminants reconnus de la beauté sont la morphologie moyenne, la culture, la numérologie et le facteur ethnique.

\subsubsection{La morphologie moyenne}

Nous connaissons tous le célèbre titre de Langlois: "Attractive faces are only average» $[103,104]$. Il est aujourd'hui reconnu que l'essentiel de la qualité esthétique repose sur le caractère «moyen» de l'objet. Ceci s'applique bien sûr au visage : un visage attirant est un visage familier, donc pour l'enfant puis l'adolescent, c'est le visage «moyen » de son environnement familial puis plus tard social [21]. La préférence pour les visages féminins [28], plus fréquents dans le paysage de l'enfant, et l'éducation expérimentale à des visages partiellement humains seulement confirment l'effet cognitif et le rôle de l'environnement dans la préférence esthétique [132, 134, 144]. En dehors du domaine maxillo-facial, la dictature de la forme moyenne s'impose partout [74].

Nos propres expériences montrent toujours des scores esthétiques favorables pour les échantillons de référence, échantillon «standard » au niveau occlusal et céphalométrique, sujets ortho-morphiques, ou pour les patients traités et donc ramenés à la norme par la thérapeutique $[51,56,57]$.

Remarquons toutefois que même Langlois ne ferme pas la porte aux facteurs autres que la morphologie moyenne $[102,103]$.

La symétrie joue un rôle majeur dans le jugement esthétique, plus certainement dans la littérature des sociologues que dans la littérature orthodontique. Sans doute parce que l'asymétrie est la plus évidente des dysmorphies à l'inspection faciale.

Les recherches récentes vont introduire deux notions complémentaires à ce sujet :

- D'une part, il semble que le normo-morphique comporte une petite asymétrie dite «naturelle», ainsi l'optimum esthétique doit prendre en compte cette asymétrie « naturelle».

- D'autre part, les asymétries sont liées aux dysmorphies antéro-postérieures, et leur condamnation pourrait être liée pour partie à la classe II/classe III associée [55, 120].

Enfin, précisons que le jugement esthétique porte sur la morphologie faciale cutanée seulement, et donc que la référence morphologique sera la morphologie faciale cutanée moyenne.

\subsubsection{La culture}

\subsubsection{Linfluence culturelle}

La culture au sens large c'est l'acquis de l'individu à travers tous les contacts de la vie sociale.

$\mathrm{Au}$ premier rang de ces influences, il faut citer les contacts sociaux effectifs (famille, cercle d'amis, environnement professionnel...) et le rôle des médias : télévision, cinéma, radio, presse écrite... Les psychologues ont étudié l'influence des émissions de télévision et des publicités sur l'image idéale $[47,115,118,136]$. 
Les modèles forts et évolutifs sont ceux surtout dictés par des possibilités étendues de morphologies possibles, la publicité et, à l'extrême, la bande dessinée. Le «casting » est beaucoup plus libre pour un spot publicitaire que pour un film et en matière de bandes dessinées, le choix du dessinateur dépend de sa seule fantaisie!

Le modèle esthétique dominant dessiné par les médias peut entraîner une adhésion servile ou au contraire une réaction de défense et de refus.

Limportance du facteur culturel intervient surtout pour l'identité sexuelle et l'identité ethnique.

\subsubsection{La recherche de la "super-beauté "}

Si toutes les études reconnaissent l'importance déterminante de la morphologie moyenne dans l'appréciation esthétique sociale, il est évident que l'idéal de «super-beauté » diffère sensiblement de la forme moyenne $[8,157]$; la poupée Barbie constitue pour beaucoup un idéal de perfection, mais force est de constater que sa morphologie corporelle ne correspond en rien à la morphologie moyenne de la jeune femme américaine.

Les études sur la «super-beauté » ont utilisé des sujets dont la carrière dépend majoritairement de leur séduction (acteurs, mannequins...) et des portraits composites. La méthode est basée sur la recherche des éléments qui différencient les superbeaux des normo-morphiques, et éventuellement sur l'accentuation des points de divergence $[36,47,118$, $122,126,157]$.

Les résultats montrent que l'attraction va dépendre de facteurs morphologiques témoignant ou semblant témoigner :

- d'ouverture ou d'expressivité,

- de jeunesse [3, 38, 97, 124, 141, 166],

- de féminité ou de sex-appeal,

- de maturité rassurante et solide ainsi que de virilité chez l'homme,

- de puissance ou de force,

- de bonne santé [71, 160, 161].

C'est ainsi que, pour un visage féminin, on préférera un nez petit (infantile), des yeux immenses (expressivité ouverture) et des lèvres pulpeuses et biprotrusives. Cunningham [28-30] montre que la préférence esthétique incline vers le petit enfant ou le bébé pour le centre du visage (nez petits, grands yeux très écartés) et vers la femme pleine de sexappeal pour les zones périphériques.

\subsubsection{La spécificité ethnique : effet morphologique ou effet culturel?}

Le rôle du groupe ethnique dans l'appréciation de la beauté est double: d'une part, la référence morphologique moyenne sera différente; d'autre part, la culture sera différente. Si l'influence de la morphologie moyenne différente est facilement appréciable ([48] par exemple), il n'en est pas de même de l'effet culturel.

Le groupe ethnique étudié peut être à vocation dominante (fierté, démographie importante, position sociale élevée...); dans ce cas, il aura tendance à accentuer pour son modèle idéal ses traits spécifiques. Le groupe ethnique peut être récessif (récession démographique, abandon de sa culture ou de sa langue...) et il aura tendance à gommer ses différences plastiques voire à adopter le modèle esthétique d'un groupe dominant. La santé d'une ethnie est liée à un idéal esthétique d'homme masculinisé [35].

Les Africains vivants en Afrique ont une préférence [52] pour les faibles biprotrusions (inférieures à la moyenne ethnique locale). Les orthodontistes afro-américains vivant aux USA préfèrent des biprotrusions marquées pour les patientes aussi afroaméricaines [75, 109], qui elles-mêmes optent pour un profil encore plus biprotrusif.

Le jugement esthétique d'une ethnie doit être le fait de l'ethnie elle-même [52]. Toutefois, dans le cas d'une société polyethnique, dans laquelle le patient, quelque soit son ethnie d'origine, doit évoluer sa vie durant, il est intéressant de différencier l'opinion des différentes ethnies composantes; il est intéressant de connaître le jugement d'un groupe sur luimême, mais aussi les jugements croisés de chaque groupe sur les autres constituants de la société. Il s'agit en Europe d'un sujet non politiquement correct. Certains chercheurs s'y sont intéressés outreatlantique $[69,75]$.

\subsubsection{La dynamique du visage est-elle génétiquement déterminée ou bien acquise?}

L'opinion générale optait pour une acquisition des mimiques liée d'abord au simple effet d'imitation de l'entourage et ensuite au désir de plaire; une origine culturelle s'imposait. Après les travaux d'Ekman, une conception darwinienne l'emporte aujourd'hui et l'ensemble des mimiques serait d'origine génétique $[45,46]$. 


\subsubsection{La numérologie}

À la suite des Arabes, la Renaissance s'est passionnée pour certains nombres irrationnels, à qui elle a prêté des valeurs magiques, voir démoniaques ou divines. Elle a aussi aimé élaborer des triangles magiques ou des carrés magiques tels que les sommes, ou produits des lignes et des colonnes ont des propriétés surprenantes. De même, les premiers nombres ont reçu une identité cosmique : chiffre de Dieu, chiffre de l'homme, chiffre de la femme...

Les irrationnels les plus connus sont $\sqrt{2}, \sqrt{3}$, e, $\pi, \phi \ldots$

Certains ont une réalité physique incontournable, par exemple $\pi$ reste le rapport de la circonférence au diamètre, c'est une obligation trigonométrique; d'autres se rapportent aux modèles cristallographiques ou physiques de base, etc.

En matière d'esthétique, particulièrement d'esthétique faciale, cette croyance dans les chiffres va conduire à la recherche permanente de critères numériques simples et universels [84, 137, 138], avec les deux domaines d'application majeurs suivants.

\subsubsection{Le nombre d'or}

Le nombre d'or, ou la «divine proportion », sont définis comme un rapport du petit au grand qui reste inchangé pour le rapport du grand au tout (ensemble petit + grand).

$$
\phi=x / y=y /(x+y)
$$

Un calcul simple montre que :

$$
\phi=(1+\sqrt{5}) / 2=1,6180
$$

\section{(c'est un irrationnel comme $\sqrt{5}$ bien sûr).}

Son appellation du nom de $\phi$ est un hommage à Phydias. Ce nombre est très lié à la suite de Fibonacci.

Son intervention dans le processus de croissance de certains crustacés est reconnue, son intervention dans l'architecture du Parthénon ou du Théâtre d'Epidaure est connue. Son rôle comme arbitre suprême de la beauté, ou du moins du rapport le plus harmonieux entre deux mesures linéaires, est soutenu par certains $[34,63,68,123,137,138,169]$.

Plusieurs remarques critiques s'imposent :

$\mathrm{Si}$ on calcule les rapports deux à deux de tous les couples de mesures possibles verticales/transversales, région mandibulaire/région frontale, orbitaire/nasale, il est inévitable de trouver des valeurs proches de $\phi$,
- Si on y adjoint comme valeurs « sacrées » $\phi^{2}, \phi^{3}$, $\phi^{4} \ldots$ et $(\phi-1),(\phi-1)^{2}$, et $(\phi-1)^{3} \ldots$

- Si on accepte une marge d'erreur de $1 \mathrm{~mm}$ ou 1 degré, ou de $5 \%$ sur les rapports,

la valeur moyenne d'un rapport quelconque peut être considérée comme sacrée!

Par ailleurs, divers auteurs ont montré que le rectangle de rapport $\phi$ n'était pas la forme préférée [34], et d'autres auteurs ont montré que, chez les Japonais, la forme préférée était de rapport $\sqrt{2}$ [116].

\subsubsection{Les canons et critères}

Les canons ou critères sont des rapports à visées normatives établis en général à partir des moyennes de certaines mesures morphologiques:

- l'auteur va modifier ce rapport selon une décision arbitraire (non justifiée sur le plan épidémiologique),

- l'auteur va imposer ce critère comme un dogme.

L'exemple type est le canon tête/corps entier de la statuaire grecque : si, à l'époque archaĩque comme en Égypte, on adopte un critère correspondant aux mesures moyennes (1/8) qui s'impose comme une règle absolue, à l'époque classique, pour donner un aspect plus monumental et pour faire des athlètes plus « athlétiques », on porte le critère à 1/9.

Les critères de ce type sont fréquents en orthodontie, on peut retenir dans ce groupe les lignes esthétiques (Ricketts, Steiner, Z de Merrifield), qui nous sont présentées comme des critères absolus d'esthétique faciale satisfaisante!

Ainsi, dans un travail de recherche sur le jugement esthétique, faudra-t-il rechercher des liens entre la note esthétique et les facteurs possibles que nous venons d'envisager, afin de comprendre mieux le processus d'appréciation.

\subsection{Déterminants indirects}

Si le jugement esthétique porte essentiellement sur la forme de l'enveloppe cutanée, les déterminants architecturaux de la face vont avoir un rôle causal et explicatif majeur : ce sont bien sûr la denture, l'architecture osseuse et l'enveloppe musculo-tendineuse.

Les orthodontistes ont trop longtemps simplement jugé de l'esthétique de leur patient par un simple coup d'œil à la téléradiographie de profil du crâne, avec un profil cutané souvent même difficile à apercevoir. La conclusion de ce bref examen, surtout des structures dentaires et osseuses, tenait en 
trois mots : «profil correct », «profil trop biprotrusif » ou « profil trop birétrusif ».

Aujourd'hui, ne tombons pas dans l'excès contraire qui consisterait à faire reposer notre diagnostic et notre plan de traitement uniquement sur un examen cutané!

Le rôle des tissus de soutien, dentaires, osseux ou musculaires, est fondamental dans la compréhension de la disgrâce liée à la dysmorphie, il est fondamental aussi dans la correction thérapeutique du préjudice esthétique, puisqu'on va déplacer ces tissus de soutien pour corriger la dysharmonie du rapport cutané

Dans le diagnostic esthétique, la morphologie cutanée est la préoccupation majeure mais une céphalométrie dento-alvéolaire, osseuse et un examen rigoureux des muscles peauciers et masticateurs au repos et en fonction sont absolument indispensables.

\subsection{Facteurs perturbateurs du jugement esthétique}

Le jugement esthétique pour l'orthodontiste doit porter uniquement sur la morphologie faciale cutanée, puisque c'est son domaine d'intervention. À l'examen d'un patient, le jugement risque d'être faussé par deux types de facteurs : les facteurs environnementaux ou périphériques et les facteurs sociopsychologiques.

\subsubsection{Facteurs esthétiques perturbateurs}

Les facteurs esthétiques «périphériques» naturels de la face sont bien sûr la chevelure, la carnation de la peau, la couleur des yeux et leur morphologie, les rides, la couleur et la texture des lèvres. Bien évidemment, les éléments cosmétiques, le maquillage, les bijoux et piercings, les lunettes, les tatouages, les teintures et décolorations, la coiffure, les éléments vestimentaires peuvent modifier encore l'aspect facial.

Le contrôle de ce problème a été obtenu de deux manières différentes au niveau des documents : lors de la réalisation des documents ou ex post par traitement «neutralisant » ces documents.

Lors de la prise des documents, il faudra veiller à éliminer les bijoux et piercing, démaquiller, tirer vers l'arrière ou cacher au mieux les cheveux [128], cacher les vêtements (col, écharpe...), standardiser les fonds ou arrière-plans et les éclairages.

Ex post, le traitement des documents par « floutage », banalisation ou neutralisation va tenter de gommer les différences de jugement que ne manquent pas d'induire les diverses textures et couleurs cutanées, par exemple. Certaines de ces techniques s'imposent déjà dans notre spécialité pour respecter la vie privée et l'image du patient. On peut classer dans cette catégorie :

- les « floutages » ou masquages des yeux,

- la substitution d'une coiffure standard aux coiffures réelles des sujets [157],

- l'utilisation de photographies en noir et blanc, soit par emploi de film noir et blanc, soit par traitement en «niveaux de gris » $[42,67,98,179]$,

- l'emploi de profils en «ombres chinoises » ou simplement de tracés linéaires $[15,31,67,75,78$, $98,152,179]$.

Certains ont même tenté de " dépersonnaliser» encore plus la détermination de l'idéal esthétique : le juge dessinera lui-même le profil idéal ou le construira comme dans un jeu de construction [42, $67,97,148]$. Des méthodes de «morphing» à partir du profil initial du patient sont aussi proposées pour apprécier l'objectif optimal de profil terminal $[66,68,79]$. Des modifications discrètes (au sens mathématique), ou pas à pas, du profil cutané peuvent être proposées par une méthode informatique interactive, à l'appréciation du juge $[65,67,68]$.

Les reconstructions des scanners ou des conebeam cranio-faciaux se prêtent parfaitement à la recherche morphologique et à la détermination de l'optimum esthétique (Figs. 1 à 4). En effet, ces reconstructions sont bien sur 3D et il suffira simplement d'orienter la tête et d'éliminer parfois une mèche rebelle pour obtenir un aspect «statue antique » du meilleur effet; et nous sommes tant habitués à apprécier la beauté en admirant la statuaire gréco-romaine!

La vraie question est : Où arrêter les retouches de «banalisation » des documents?

Les critiques suivantes tendent à limiter notre enthousiasme pour ce type de documents.

- D'une part, en déshumanisant à l'extrême le document, par exemple en faisant un choix de profil parmi une série de tracés au crayon, noir sur fond blanc, est-on vraiment sûr d'être mis dans les conditions réelles de l'appréciation sociale quotidienne qui nous fait dire, regardant notre voisine dans le métro: «Cette jeune femme est vraiment très jolie »?

Je ne le crois pas. 


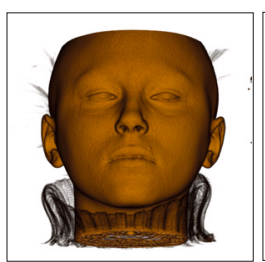

a

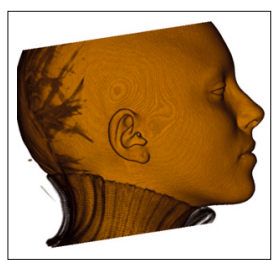

b

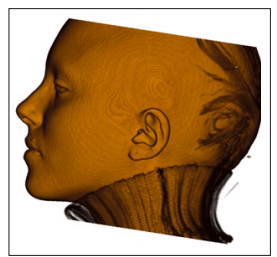

C

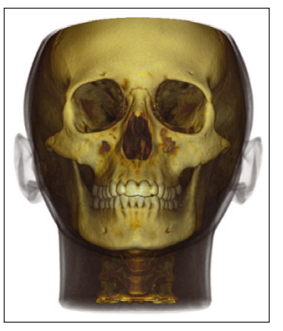

d

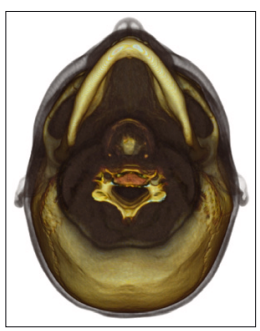

e

Figure 1

La qualité du rendu cutané et osseux. À partir d'une saisie RX, ici scanner, il est possible de réaliser une reconstitution cutanée avec le rendu esthétique d'une statue de marbre $(\mathrm{a}-\mathrm{c})$ et une reconstitution des tissus durs avec un excellent résultat et un « rendu réaliste » évoquant les squelettes secs de nos laboratoires d'anatomie (d-e).

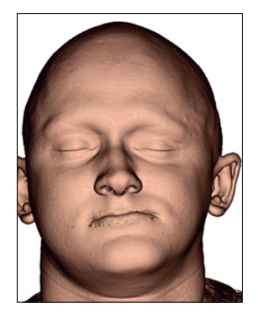

a

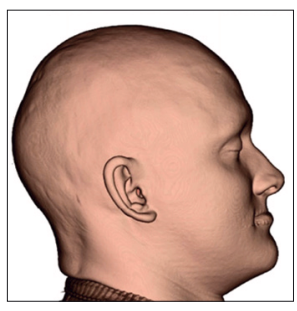

b

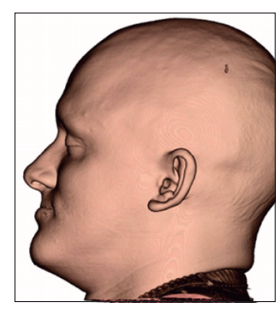

C

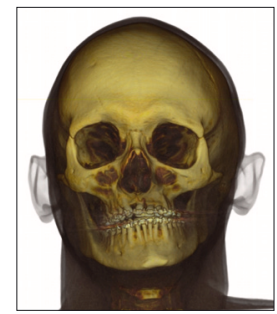

d

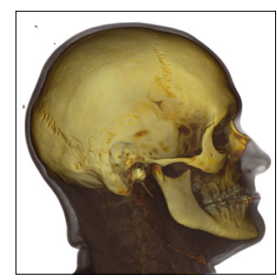

f

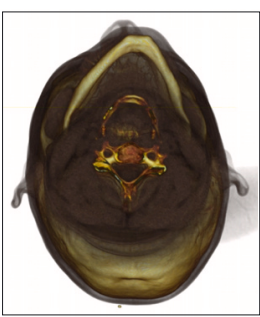

e

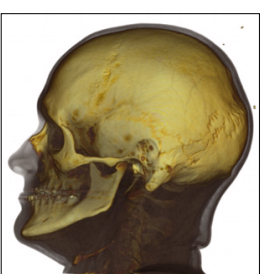

g

Figure 2

La reconstitution et le diagnostic de la dysmorphie. La reconstitution cutanée (a-c) et la reconstitution osseuse parallèle, avec les tissus mous en transparence $(\mathrm{d}-\mathrm{g})$, permettent de préciser le diagnostic. Dans ce cas, l'examen de l'architecture osseuse permet de préciser l'importance et la localisation de la dysmorphie. La seule inspection visuelle n'évoquait pas ici une asymétrie par excès de l'hémi-mandibule droite aussi importante et avec des composantes verticales et antéro-postérieures majeures. 


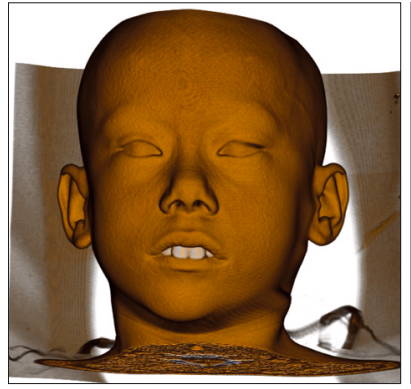

a

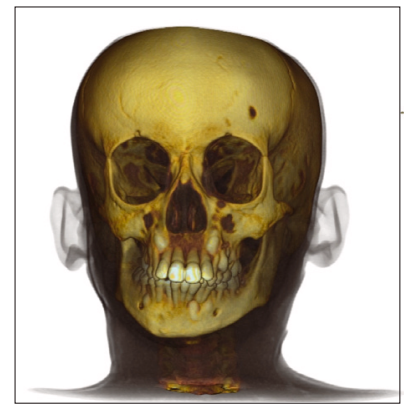

d

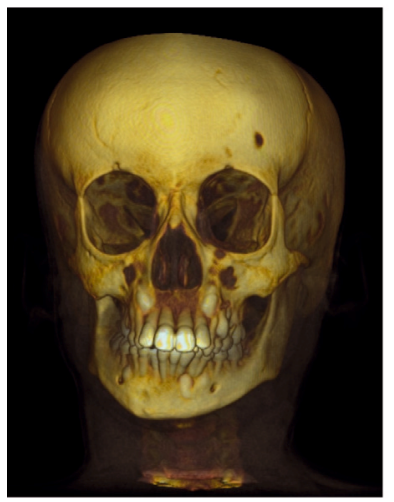

g

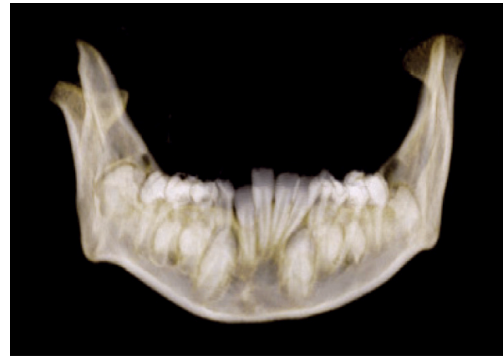

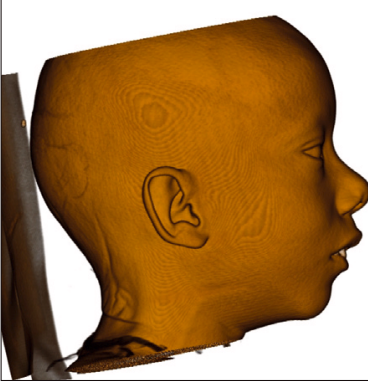

b
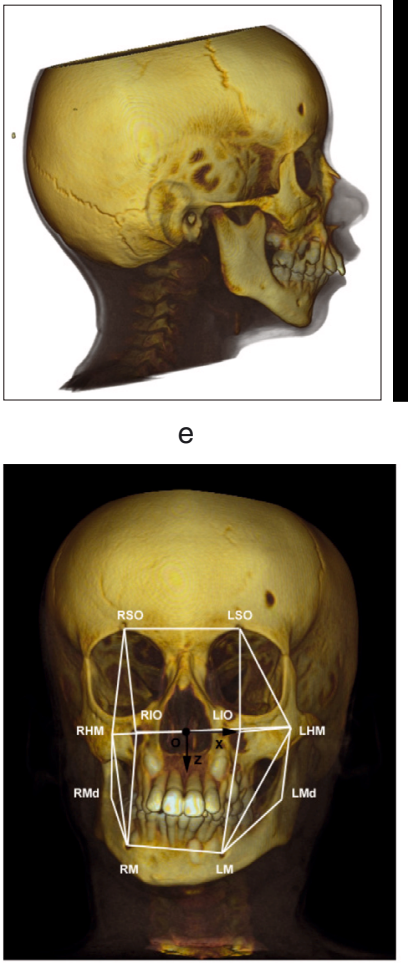

h

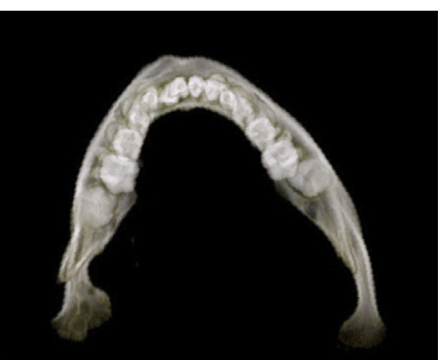

$\mathrm{k}$

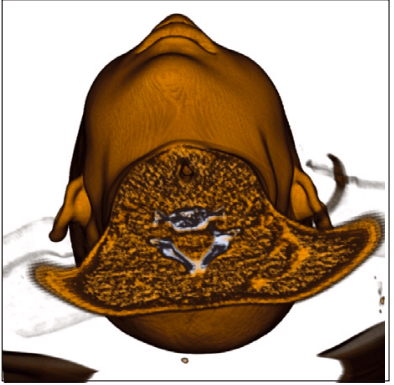

C

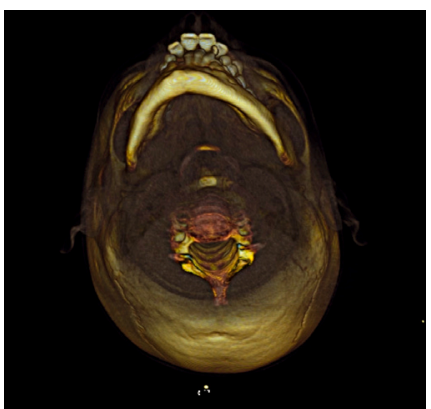

f

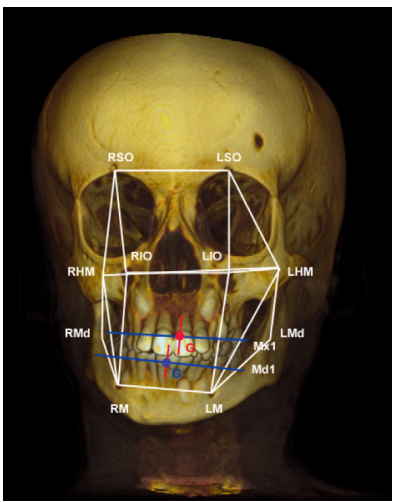

i

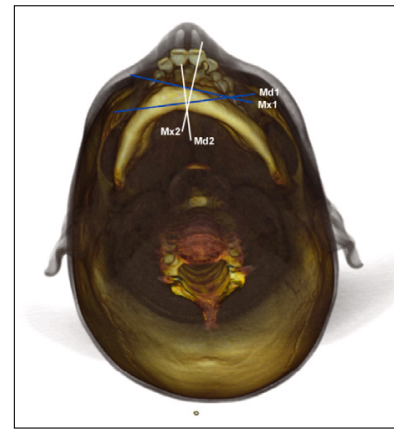

I

Figure 3

Vers le diagnostic céphalométrique complet. Chez ce patient dont la classe II,1 hyper-divergente s'impose dès l'inspection cutanée (images avec béance labiale découvrant les incisives (a-c)) ou squelettique (d-f), la céphalométrie osseuse va préciser le diagnostic. $(\mathrm{g})$ et $(\mathrm{h})$ montrent la mise en place des 14 points repères trigéminaux, du cadre ou enveloppe maxillo-faciale, enfin du référentiel orthonormé $(\mathrm{Ox}, \mathrm{y}, \mathrm{z})$. (i) et (j) montrent la mise en place des axes d'inertie d'arcades (axes d'inertie maxillaire et mandibulaire (rouge et bleu), avec une bascule horaire dans le plan frontal. (k) et (I) montrent la rotation dans le plan d'arcade des axes d'inertie des arcades ( $\mathrm{Mx}$ et $\mathrm{Md}$ : maxillaire et mandibulaire ; axe 1 et axe 2, respectivement transversal et postéro-antérieur). 


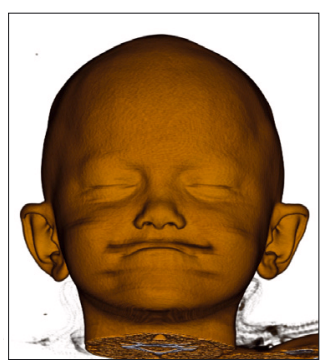

a

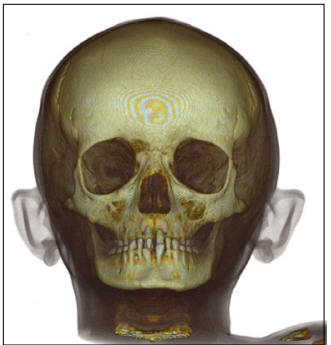

d

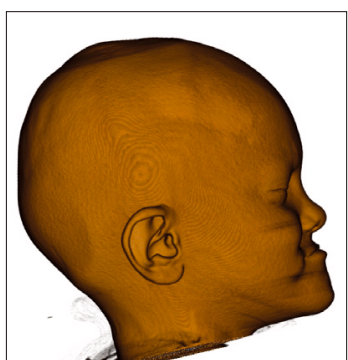

b

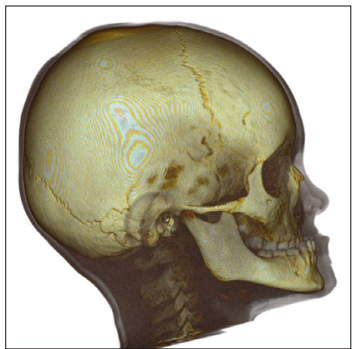

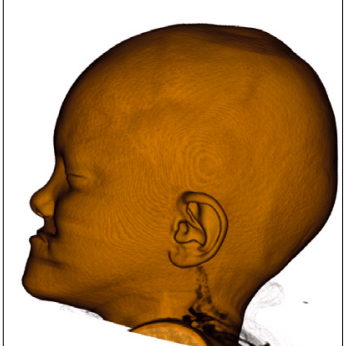

C

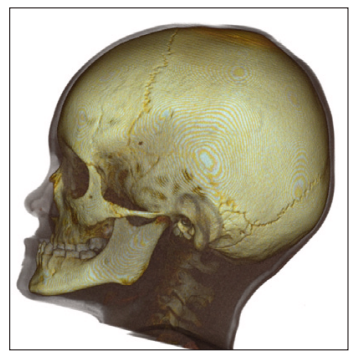

f

Figure 4

Au-delà des descriptions cutanées et osseuses précises : la mimique. Chez ce patient en classe III hypodivergente, probablement chirurgicale, l'aspect facial grimaçant témoigne de la difficulté ou même de l'impossibilité d'une mimique apaisée. On évoque irrésistiblement un buste de Messerschmidt.

- La seconde critique est plus subtile : certains des déterminants esthétiques non morphologiques sont indirectement liés à la morphologie cutanée; une biproalvéolie est souvent liée à une peau sombre ou des yeux bleu clairs à une carnation très claire aussi et une birétroalvéolie. En éliminant l'information sur les yeux ou la texture cutanée, on détruit un ensemble harmonieux!

La seconde manière d'éliminer l'influence des facteurs perturbateurs est statistique; en ayant des échantillons beaucoup plus étendus, l'effet attractif (ou répulsif) d'une longue chevelure (ou coiffure courte) ne perturbera pas, par exemple, une étude sur le préjudice esthétique associé aux dysmorphies antéropostérieures; les différents types de coiffure seront également répartis entre les dysmorphies; un test statistique permettra même de contrôler l'indépendance des deux choses.

\subsubsection{Facteurs socio-psychologiques}

Divers facteurs tenant à la personnalité globale du sujet viennent encore perturber l'appréciation thérapeutique maxillo-faciale pure : une personnalité brillante, un discours plein d'humour, une attitude assurée, une aisance sociale inciteront le juge à émettre inconsciemment une opinion plus favorable sur la morphologie maxillo-faciale, objet du jugement demandé.

Ces facteurs concernent l'ensemble de la personnalité du sujet et s'étendent des domaines purement physiques jusqu'à des domaines purement intellectuels : une silhouette bien droite et décidée sera mieux cotée qu'un corps tout voûté ; un intellectuel brillant, à la conversation pleine d'humour, jouira d'une sur-notation liée à son aura naturelle. Les sociologues retiennent les facteurs généraux suivants :

- Lintelligence : la relation intelligence/beauté est à double sens ; celui qui est beau est présumé intelligent et celui qui est intelligent gagne en cotation esthétique [90].

- La symétrie : beaucoup d'auteurs mettent en évidence le rôle de la symétrie dans la préférence esthétique [107, 110, 125, 135].

- Le dimorphisme sexuel : chez l'homme, par exemple, les éléments déterminants seront la posture fière et droite, le torse athlétique, la musculature, plus encore semble-t-il pour des juges masculins que féminins $[60,64,97,125]$.

Chez la femme, ce sera bien sûr la poitrine, les hanches ou les fesses qui joueront un rôle majeur. 
Si le dimorphisme sexuel influence fortement le jugement, bien sûr cela dépendra du sexe ou de l'orientation sexuelle du juge [70]. Chez les femmes, la préférence esthétique est en outre variable en fonction du cycle $[12,15,127]$.

- La silhouette : les paramètres corporels généraux tels que WCR, WHR, LBR et BMI (Waist to Chest Ratio, Waist to Hip Ratio, Leg to Body Ratio, Body to Mass Index [64, 153]) jouent un rôle déterminant. Les valeurs idéales de ces paramètres varient beaucoup selon le pays. Pour le WHR par exemple, il évolue chez la femme de 0,6 en Chine pour atteindre 0,8 à 0,9 en Amérique du Sud ou en Afrique [40, 61, 62].

Le rapport longueur des jambes/taille globale est particulièrement déterminant surtout pour la femme $[14,155,156]$. Le rôle des pieds même est non négligeable dans l'esthétique globale [177].

- La taille : chez l'homme, elle est toujours un élément positif. Mais elle joue aussi un rôle déterminant pour la femme, chez qui une taille inférieure à celle de son conjoint est toujours souhaitée. Certains résultats montrent que les femmes de petite taille sont considérées comme meilleures reproductrices.

- La chevelure et la pilosité : chez l'homme, un système pileux développé est considéré comme un élément défavorable. Mais, dans certaines zones géographiques, une pilosité modérée est préférée à une absence total de poils [39-41].

- La texture et la coloration cutanées : d'une manière générale, des peaux claires sont préférées chez la femme tandis que des peaux sombres sont préférées chez l'homme. Des peaux plus rouges ou plus jaunes attirent par l'impression qu'elles donnent de bonne santé [142, 159-161].

- Les odeurs corporelles : diverses études en double aveugle montrent une préférence des femmes pour l'odeur des hommes de face harmonieuse et symétrique [133, 139].

- Lâge : l'attrait de femmes jeunes pour le jury masculin est nettement lié au potentiel reproductif qu'elles peuvent offrir, ainsi qu'à la masculinisation de l'aspect facial avec la modification du rapport oestrogènes/androgènes [38, 168, 182].

Les facteurs que nous venons d'étudier sommairement trouvent leur source directement dans des données psychologico-sociales ou bien dans des données physiques liées à une charge socio-psychologique forte. Ils paraissent a priori fort distants de notre sujet d'étude et de notre domaine d'intervention, la morphologie faciale; mais nous avons vu qu'ils sont susceptibles d'intervenir fortement dans le jugement esthétique ou d'introduire des biais dans le traitement statistique. Ainsi, une étude sur le préjudice social des dysmorphies antéropostérieures devrait affecter la note esthétique d'une pondération selon l'âge des sujets pour corriger le biais lié à la préférence pour les sujets les plus jeunes (ou bien recruter un échantillon d'âge homogène).

Si ces facteurs peuvent être éliminés sans trop de mal dans une étude scientifique (suppression de la pilosité apparente, odeur bien sûr non rendue par la photographie, silhouette générale non restituée par le document photographique...), ils ne peuvent l'être lors du diagnostic clinique, où le praticien est en contact physique avec son patient : un patient de classe II chirurgicale pourrait ainsi échapper à une orientation thérapeutique ortho-chirurgicale parce que sa silhouette jeune et sportive, son sourire facile, son contact sympathique gomment en partie l'impression négative apportée par la classe II importante.

\section{Les moyens de l'appréciation esthétique sociale}

Lappréciation esthétique dans un but thérapeutique (diagnostic, suivi, critique du résultat, simulation...) doit associer un jugement quantifié, une note, à une description morphologique elle-même quantifiée.

Nous traiterons donc ici des modalités de jugement et des analyses de forme associées à l'objet étudié.

\subsection{Modalités de jugement}

Les modalités pratiques de l'appréciation esthétique dépendent bien sûr surtout du but poursuivi : simple diagnostic ou objectif de recherche orienté vers un champ précis. Le protocole diffère ainsi à différents niveaux : choix des échantillons, nature et traitement des documents, conditions de notation, nature du jury.

\subsection{1. Échantillon}

Léchantillon d'étude peut être réel ou virtuel. 


\subsubsection{1. Échantillon réel}

On peut étudier une pathologie ou une évolution thérapeutique à partir des documents de patients ayant vraiment cette pathologie ou ayant effectivement profité de la thérapeutique étudiée $[12,15,16$, $42,52,57,75,82,86,111,128,129,152,176]$.

\subsubsection{2. Échantillon virtuel}

On peut aussi employer les documents de sujets ayant des visages normo-morphiques et déformer les documents par des techniques de "morphing»; on parvient ainsi à simuler la dysmorphie et même son traitement $[66,68,77,79,80,87,99,143,157]$.

Avec des échantillons naturels, il faut neutraliser l'effet des facteurs esthétiques perturbateurs (non liés à la morphologie : par exemple la texture cutanée, la couleur des cheveux) et des facteurs sociopsychologiques (par exemple l'aura, la silhouette). Leffet de paramètres non pris en compte dans l'étude devra aussi être éliminé (par exemple l'état gingival dans une étude sur la forme faciale).

Ceci peut être réalisé soit par des traitements de « banalisation » $[15,75,152]$, ou en élargissant l'échantillon à l'aide de clones virtuels pour lesquels les éléments capillaires ou cosmétiques ont été modifiés [157]. On peut aussi élargir l'échantillon en vue de neutraliser ces effets pervers.

Dans le second cas, l'étude peut très bien n'utiliser que peu de patients voire même un seul, déformé progressivement pour simuler les différents degrés de dysmorphie ou les différents résultats thérapeutiques possibles.

\subsubsection{3. Échantillon composite}

La solution qui permet d'économiser l'attention de juges est la création de stéréotypes par fusion d'une série de portraits de patients présentant une typologie voisine pour réaliser un portrait composite. Cette méthode mise au point par Galton en 1878 est bien facilitée aujourd'hui avec les photographies numériques et est souvent utilisée [13, 43, 103, 104, 126, 144, 157]. Elle a l'inconvénient évident de cette manie très $19^{e}$ siècle des stéréotypes dont elle est issue (liée à la morphopsychologie : tête d'assassin, tête de voleur, tête de délinquant...) : elle crée artificiellement des sous-groupes dans une population gaussienne.
On peut critiquer cette méthode parce que, pour certains auteurs, les portraits composites sont rendus plus attirants par le nécessaire traitement technique (normalisation du contraste, harmonisation d'échelle, en général sur la distance bi-pupillaire, protocole de fusion... [8]), mais ce n'est pas l'opinion dominante [79].

\subsubsection{4. Échantillon de référence}

L'emploi d'un échantillon de référence est en général nécessaire pour donner la note moyenne autour de laquelle se différencieront les « beaux » et les «laids» $[15,51,56]$.

Ceci pose un difficile problème de sélection :

- Faut-il sélectionner des sujets au hasard simplement avec âge, sexe et origine ethnique identiques à ceux de l'échantillon d'étude (population globale)?

- Faut-il sélectionner des patients non traités avec une occlusion parfaite, et il y en a peu?

- Ou bien peut-on accepter comme idéal un lot de patients traités avec une occlusion parfaite, confortant par-là la décision du praticien traitant?

- Faut-il que les sujets normaux soient soumis d'abord à un critère occlusal (classe I parfaite et absence de problème ATM) et ensuite à un critère de normalité céphalométrique?

Lintervention d'un échantillon de référence reste toujours nécessaire même lorsqu'on étudie une variation esthétique avant/après : si des tests appariés permettent de connaître la réponse (variation positive ou négative chiffrée), il est utile de savoir si l'aspect avant ou l'aspect après sont pénalisés par rapport à la normalité. Par ailleurs, l'échantillon de référence permet d'apprécier le comportement du jury $[50,51,57]$.

\subsubsection{Documents d'appréciation}

\subsubsection{Documents classiques (statiques)}

Le choix des documents de travail dépend bien sûr de l'objectif de la recherche : esthétique faciale globale, esthétique bucco-dentaire, esthétique dentaire seulement? Deux choix essentiels s'imposent : l'angle d'observation et la zone d'observation (le champ). 
Les documents complets comprennent classiquement : les portraits ( 3 ou 4), les vues dento-labiales (sourire: parfois seulement la vue de face), enfin les trois vues endo-buccales vestibulaires.

\section{- Les portraits}

Longtemps les orthodontistes ont privilégié l'observation du profil, très influencés par les documents radiologiques qu'ils manipulent le plus souvent, les téléradiographies du crâne de profil; les publications font alors référence à «l'esthétique du profil »[36, 67, 75, 80, 98, 111, 130, 152, 157]. La préférence pour le profil est liée à la disponibilité permanente du film téléradiographique profil-crâne et à la prédominance, en orthodontie comme en chirurgie, des actions antéropostérieures, supposées plus lisibles sur les vues de profil [51].

Pourtant, dans la vie sociale, la séduction d'un visage s'apprécie surtout de face puisque l'on regarde en règle générale son interlocuteur de face. La vue de trois quarts, privilégiée par les peintres portraitistes de la Renaissance, synthétise les aspects face et profil et semble plus vivante, moins figée; mais elle ne se prête pas aux mensurations!

En fait, les appréciations sur les portraits de profil, de face ou de face souriant sont homogènes $[51,57]$, avec simplement une sévérité accrue pour la note du profil [51].

Pour avoir une vue plus globale de l'esthétique faciale, certains auteurs réunissent deux ou trois photographies (face, profil et trois-quarts) sur un même document $[42,52,56]$; certains même présentent simultanément quatre documents (face repos, face sourire, profil, trois quarts [128]).

\section{- Les vues dento-labiales (sourire)}

Lorsque l'on s'intéresse particulièrement aux dents ou aux lèvres, il est intéressant de cadrer la photographie sur la zone considérée de manière à éliminer l'influence perturbante du reste du visage $[23,50,86,99,130,140]$. Mais les éléments d'un visage sont interdépendants, sur le plan anatomique et sur le plan physiologique, comme aussi sur le plan esthétique. En portant un jugement in abstracto sur l'aspect d'une denture antérieure à partir de la vue endo-buccale de face ou des moulages, on néglige délibérément le rôle prédominant des lèvres qui cachent certaines dents, portent des ombres sur d'autres, harmonisent ou non leur forme et leur texture avec celles de la gencive et des dents. L'emploi de photographies de détails (par exemple recadrées autour de la bouche) est intéressant, mais il doit nécessairement, pour nous, être complété par celui des photographies d'ensemble du visage [50].

Ces vues du sourire sont difficiles à réaliser et posent plusieurs problèmes :

1. Ce sont des tentatives pour caractériser un phénomène dynamique, par un document statique.

Où arrêter le sourire?

Pour beaucoup d'auteurs, la référence est le sourire le plus large possible avant le rire (c'est la définition physiologique). D'autres auteurs distinguent le «sourire naturel» auquel succède le "sourire forcé » : ce serait le premier et surtout pas le second qu'il faudrait considérer [146, 154] !

Sur le plan technique, l'emploi d'un appareil motorisé prenant des photographies « en rafale » est souhaitable voire indispensable.

2. Pour la plupart des auteurs, la photographie de face suffit, mais certains travaillent sur le rôle esthétique de la canine dans le sourire et utilisent des photographies-sourires de profil ou de trois quarts.

Les travaux consacrés au sourire bucco-dentaire et basés essentiellement sur les photographiessourire (champ restreint ou champ moyen) :

- s'intéressent prioritairement aux défauts labiaux, dentaires ou prothétiques [50,99, 154],

- s'intéressent surtout à la thérapeutique (orthodontie, dentisterie cosmétique, implantologie, prothèse...) [1, 58, 59, 87, 88, 95, 146, 154, 169],

- cherchent souvent à définir des critères, clés ou diagrammes de beauté du sourire, soit par l'étude des lignes du sourire, soit par l'étude des relations chiffrées (rapports numériques) entre les dimensions des dents, des lèvres, de la gencive exposée, du corridor buccal... [1, 10, 23, 58, 59, 113, 141, $154,167,169]$.

Ces travaux reposent en général sur des mesures morphologiques et l'opinion d'un jury fait le plus souvent défaut.

\subsubsection{Traitement des documents}

La «banalisation » des documents est l'élimination nécessaire des éléments esthétiques perturbateurs d'un jugement qui se veut purement basé sur la morphologie, tels que texture cutanée, coloration cutanée, couleur des cheveux et coiffure, maquillage... Ces éléments ne doivent pas être pris en compte par 
l'étude puisqu'ils ne dépendent pas de l'action de l'orthodontiste.

Nous avons étudié plus haut l'intervention de ces éléments esthétiques perturbateurs, et précisé la manière de les contrôler :

- soit par «banalisation » des documents photographiques au niveau des prises de vues ou ex post par retouche d'élimination des détails perturbateurs (bijoux, couleur des cheveux...),

- soit en disposant d'échantillons plus larges.

\subsubsection{Les documents $3 D$}

Les documents tridimensionnels ont fait une apparition récente parmi les possibilités de restitution de l'image 3D de l'enveloppe cutanée de la face, dans un but d'appréciation esthétique; simultanément, la reconstitution 3D de la denture et du squelette s'est développée pour des raisons architecturales [25, 26, $37,54,76,83,91,92,94,95,112,147,164,165,180]$.

Lidéal serait avec une seule saisie de disposer des informations topographiques de haute qualité, sur le squelette, la denture et l'enveloppe cutanée, et de pouvoir ainsi avoir une appréciation esthétique sur les deux dernières et une restitution précise de l'occlusion et de l'architecture osseuse.

La difficulté est que les saisies scanner conventionnelles ou les saisies cone beam, aujourd'hui préférées, sont souvent insuffisantes au niveau dentaire comme au niveau cutané (défaut de résolution et pas d'information de couleur).

Cela a conduit les différents auteurs à utiliser des saisies séparées pour la denture et pour la « coquille » cutanée, en conservant la saisie scanner ou conebeam pour le squelette.

Nous envisageons ici ces méthodes de saisies dentaires et cutanées, sachant qu'elles posent un autre problème délicat : le rapprochement, «la fusion » de ces saisies séparées au sein de la saisie squelettique.

\section{- Méthode de saisie cutanée ou occluso-dentaire}

Pour la saisie de la «coquille» cutanée, et plus accessoirement de la denture, les procédés actuellement disponibles sont [92] :

- La photogrammétrie [25] : dérivée des principes de la stéréographie largement utilisée en cartographie depuis très longtemps.
- Le scanner optique [25] : la saisie par scanner optique permet un enregistrement 3D de la «coquille» cutanée avec restitution des couleurs.

- La lumière structurée : un éclairage structuré (diagramme), projeté sur la surface de l'objet étudié est déformé. Lanalyse de la déformation permet la connaissance de la surface.

- Les techniques radiographiques: scanner et conebeam. Ces techniques ne donnent pas bien sûr un rendu de couleurs naturelles, et présentent en général une résolution insuffisante au niveau occlusal notamment. Certains auteurs font une saisie dentaire séparée haute résolution [164]. Remarquons que les documents scanner haute résolution, qui ressemblent à des statues de marbre, se prêtent parfaitement au jugement sur l'esthétique de la forme faciale : l'élimination de la carnation, de la couleur des yeux, des cheveux n'est plus à faire!

Nous adoptons pour notre part une saisie RX avec un protocole autorisant simultanément une biométrie 3D précise et une reconstitution tridimensionnelle de résolution correcte (Figs. 1 à 4).

D'autres moyens ont été envisagés : imagerie IRM, ultrasons 3D, holographie.

\section{- Méthodes de "fusion"}

La fusion du dentaire ou du cutané dans l'architecture squelettique apparaît comme une nécessité première; il y a un lien évident entre les modifications de forme dentaire ou squelettique (thérapeutique orthopédique, chirurgie ou orthodontie) et l'aspect cutané; aussi une simulation d'un résultat cutané implique a priori la connaissance du déplacement thérapeutique osseux ou dentaire; mais certains auteurs effectuent leurs recherches, leurs analyses et leur simulations en ne prenant en compte que la «coquille» cutanée $[91,94,147]$; ce qui est étudié c'est l'évolution de la coquille par rapport à elle-même.

De la même manière, la superposition de saisies de la denture à des temps différents permet de lire les mouvements relatifs des dents. Mais, dans un rapprochement du groupe incisivo-canin et des groupes molaires, il faut bien savoir s'il y a eu recul incisivocanin ou perte d'ancrage molaire, et donc disposer d'un repère cranio-facial! 
D'autres auteurs superposent sur les zones stables du squelette (base du crâne ou orbite) et mettent ainsi en évidence l'évolution du cutané ou de la denture dans ses rapports avec l'architecture : une méthode de fusion est alors indispensable. Diverses solutions ont été proposées [174,175], dont certaines fort compliquées [175].

La fusion difficile des structures dentaires et cutanées, saisies par exemple par une méthode optique, au sein de la saisie squelettique, élément stable, permet seule la simulation, avant, ou le suivi, après. Au niveau orthodontique, il serait intéressant de pouvoir répéter fréquemment ces saisies dentaires ou cutanée pour suivre la thérapeutique mois par mois, mais ceci appellerait alors une répétition aussi fréquente et donc impossible des saisies squelettiques RX.

\subsubsection{Les documents dynamiques}

Un visage s'apprécie surtout lorsqu'il sourit, c'est pourquoi le premier geste de contact social, d'abord chez le nourrisson, est le sourire. Si nous jugeons des visages sérieux, parfois tendus, toujours figés voir tristes, nous n'avons qu'une idée très limitée de l'esthétique faciale! Certaines dysmorphies, par exemple un grand excès vertical, perturberont plus le sourire, la mimique étant gênée par l'effort constant de tension labiale, que l'aspect au repos où le contact réussit néanmoins à être présent. Dans certaines dysmorphies, le caractère inesthétique s'accentue même avec le sourire (paralysies faciales unilatérales). L'examen du sourire est donc fondamental.

La réalisation de portraits «sourire » soit de l'ensemble du visage, soit en champ limité à la zone sous-nasale, constitue un premier pas dans le sens de cette analyse; rappelons toutefois les problèmes de champ, d'angle d'observation (face, trois quarts ou profil) et de limite du sourire à photographier; pour la plupart c'est le sourire le plus ample, avant le sourire forcé $[3,4,8]$.

La majorité des auteurs réunit dans un même document les photographies statiques et les photographies du sourire. Le cadrage étroit de la bouche souriante ne doit en effet pas être utilisé isolément, il constitue un document intéressant complété par l'aspect facial global.

Il est possible aujourd'hui d'utiliser pour juger des documents animés [2, 145], exigeant un équipement technique particulier et beaucoup de temps pour les réalisateurs comme pour les juges.

Les documents vidéo 3D sont disponibles pour l'étude des mouvements masticatoires ou des mimiques. L'emploi de repères cutanés permet les suivis dynamiques $[24,73,170,171]$.

\subsubsection{Jugement}

\subsubsection{Composition du jury}

Trois orientations intéressantes ont été évoquées :

- Jury susceptible de représenter au mieux l'opinion esthétique du groupe social dans lequel vit le patient.

- Jury de professionnels de l'esthétique ou, mieux, de thérapeutes à visées esthétiques.

- Auto-jugement.

La majorité des articles scientifiques préfère à juste titre le jury populaire, le jury « d'hommes de la rue », mais beaucoup jugent plus prudent de doubler cette opinion par celle d'un jury de professionnels de l'art ou de la santé $[12,27,42,75,80,82,87,99,128,130$, $143,152,157,166,176]$. Est-ce une trace du système bicaméral qui veut modérer la jeunesse fougueuse de la chambre basse par la sagesse de la chambre haute? Est-ce une influence des cours d'assises françaises ou la décision procède conjointement de l'opinion du jury populaire et de la sagesse des juges professionnels?

Certains se contentent à tort du jugement des professionnels [181].

Il nous semble que vouloir limiter le rôle de juge esthétique à des professionnels impliqués dans l'esthétique (artistes, étudiants des Beaux-Arts [20,179], coiffeurs, architectes, esthéticiennes, artisans ébénistes ou céramistes, décorateurs, stylistes, chirurgiens plasticiens [181], chirurgiens-dentistes...) relève surtout d'un préjugé de classe! Deux questions se posent :

- L'homme de la rue est-il vraiment incapable d'avoir une émotion artistique, d'apprécier une œuvre d'art, ou la beauté d'un vêtement, d'une automobile...?

- Comme nous l'écrivions en 2004 : « Le patient souhaite-t-il plaire à sa petite amie ou bien à son dentiste?»

Si nous sommes persuadés de la prééminence du jugement populaire ou social sur l'opinion du professionnel, cette dernière a néanmoins un intérêt 
lorsque la question posée implique une compréhension de la thérapeutique : qui pourrait mieux comprendre les remaniements cutanés dans une chirurgie de fente par exemple et, par conséquent, mieux apprécier le changement esthétique qu'un chirurgien maxillo-facial $[12,42,111,128]$ ? Pour saisir le remaniement morphologique lié à un recul incisif important, l'orthodontiste est qualifié [181]. Pour des travaux cosmétiques dentaires qui comprendra mieux le lien thérapeutique-esthétique que le chirurgiendentiste $[31,87,99]$ ?

Par ailleurs, dans bien des circonstances, les jugements professionnels ou populaires donnent des résultats homogènes. Dans ce cas, dans l'action thérapeutique, le praticien pourra se contenter de son appréciation personnelle, toujours disponible.

Lauto-jugement a été évoqué ou même utilisé par certains auteurs, de manières différentes :

- appréciation d'une dysmorphie et de son amélioration par des sujets porteurs de la même dysmorphie $[12,67,108,128]$;

- jugement familial [129,150,151], jugement porté sur le patient par sa famille: frères et sœurs, parents...;

- jugement porté sur lui-même par le patient [106].

Ces méthodes se rejoignent en fait puisque nous savons combien les grandes dysmorphies sont souvent familiales.

D’une manière générale, le jugement porté sur sa propre dysmorphie est plus clément pour les grandes pathologies, mais plus sévère pour les pathologies modérées [56, 98].

Pour les psychologues, l'auto-appréciation serait à mi-chemin entre le schéma social idéal, la morphologie réelle du sujet et la vision idéalisée du sujet par lui-même [69, 104].

Une autre manière indirecte d'introduire un autojugement est le rapprochement de la dysmorphie quantifiée, avec le ressenti exprimé par le patient du besoin de traitement, ou bien l'acceptation du traitement par exemple chirurgical, ou encore la critique positive du résultat $[3-6,72,81,85,131,135]$.

La composition du jury mérite d'être discutée : faut-il sélectionner un jury resserré et homogène, par exemple un groupe d'hommes mûrs, mariés, de situations sociales aisées, avec une fourchette d'âges de 35 à 45 ans? Non bien sûr, il faut essayer de couvrir l'ensemble de la société : âges répartis, sexes également représentés, origines ethniques diverses, niveaux culturels différents, etc.

Toutefois, les résultats montrent que les jugements portés sont globalement homogènes [50, 52, $57,163]$, même lorsque les jurys ont une composition très hétérogène en âge, en sexe, en situation... Strzalho et Kaszycka [163] ont même quantifié la part de la note relevant d'une communauté d'appréciation et la part relevant du comportement individuel du juge. Ceci justifie la notion d'« esthétique sociale $»$.

\subsubsection{Modalités de jugement}

Les modalités pratiques de jugement supposent des règles strictes pour la présentation des documents, la nature de la question posée, les modalités du jugement et les moyens d'expression du verdict.

Les documents sont rendus anonymes dans la mesure du possible, mais on peut présenter les photographies séparément $[15,50,57]$, ou bien les grouper sur un même document $[12,42,52,56,128]$.

Le regroupement sur un document unique de photographies du même sujet, prises sous des angles différents, ou avec des champs différents est fréquemment utilisé, il permet d'avoir une représentation plus complète de la face, soit par un pseudo-3D, soit par un pseudo-travelling:

- profil droit, vue de face, profil gauche,

- arcades dentaires vues de face, sourire buccodentaire vu de face, portrait souriant vu de face.

Le regroupement de documents de dates différentes est à proscrire $[42,80]$; la présentation, par exemple, des portraits de face, de trois quarts et de profil avant et après chirurgie orthognathique, associée à une question sur l'amélioration esthétique, introduit un biais évident : le juge est induit à trouver une amélioration ou une dégradation selon son opinion préalable $[80,98]$.

La nature de la question posée est un autre sujet de débat. Certains posent une ou plusieurs questions complexes, portant sur la thérapeutique, induisant l'idée imposée d'une amélioration, se recoupant dans leurs réponses...

La meilleure question doit être la plus simple et porter uniquement sur l'esthétique : le patient présenté en portrait de face ou sur un groupe de photographies (profil droit, face, profil gauche), est-il beau ou laid? S'il y a une amélioration ou si celle-ci est due 
à la croissance nasale : c'est au statisticien de l'établir. Trop souvent, on demande au juge une indication de traitement [12, 129], une évaluation de l'évolution thérapeutique directe ou indirecte $[12,16,129,162]$.

Les conditions de jugement doivent être aussi strictement codifiées.

Ceci suppose des délais d'oubli et des conditions d'isolement.

Un jugement sur l'évolution esthétique des visages entre 15 et 25 ans nécessitera une notation séparée des deux groupes de photographies, sans que le juge puisse reconnaître tel ou tel visage. Il faut donc ménager des temps d'oubli.

Le juge, pour la plupart des auteurs, doit s'exprimer isolément.

Toutefois, cette idée peut être contestée : ce que l'on souhaite est une opinion sociale, et le jugement de chacun est influencé par l'opinion du voisin. Ne faudrait-il pas favoriser un dialogue au sein du jury réuni, pour obtenir un jugement consensuel, comme cela se fait en cour d'assises?

La dernière question est le mode d'expression de l'opinion du juge : jeu limité d'appréciations, note, rang de classement.

- Certains pensent que le juge ne peut, en matière d'esthétique, donner une appréciation très fine, par exemple une note sur 10 ; ils se contentent donc d'une variable aléatoire de type "catégorie » avec 2, 3 ou 5 « cases». Bien évidemment, le traitement statistique d'une variable-catégorie avec un nombre restreint de "cases » imposera l'utilisation de pourcentages et donnera des résultats moins précis qu'une variable aléatoire continue de type note. En multipliant le nombre de « cases », on rapproche la variable catégorie d'une variable continue de type note; c'est ainsi que l'on retrouve dans les recherches sur l'esthétique faciale des variables avec 2 à 7 classes [12, 15, 86, $143,152]$.

- D'autres encore refusent la rigidité des notes et proposent de demander un classement $[50,57]$. Cette méthode semble plus proche de la démarche psychologique de jugement individuel, qui repose toujours sur des comparaisons deux à deux; elle a l'avantage d'éviter les «flous» de notation pour les sujets moyens : lorsque le juge qui a apprécié sans hésiter les sujets extrêmes, fatigué, met une note standard au « ventre mou » des sujets «moyens » (encore faut-il interdire les ex aequo!) ; cette méthode présente deux inconvénients majeurs : elle impose l'emploi de statistiques d'ordre (difficiles et moins précises) et elle limite l'étendu de l'échantillon : il est difficile de classer rigoureusement plus de 20 ou 30 sujets.

- La majorité des auteurs utilise une note esthétique entre 0 et $10[51,53,56,75,80,82,87,99$, $111,128,157]$. Elle est obtenue à l'aide d'un curseur, que le juge fait glisser sur un rail, entre les limites extrêmes «très laid» et «très beau ». La position du curseur est lisible sur une échelle millimétrée à l'envers de la fiche, ou enregistrée par une méthode informatique : il en résulte une notation précise.

\subsubsection{Comportement du jury}

La connaissance des variations subtiles de l'opinion du juge selon son sexe, son âge, son origine sociale, sa profession, son appartenance ethnique... est nécessaire afin de composer le jury optimal pour la mission d'estimation envisagée, c'est-à-dire un jury représentatif du groupe social dans lequel évolue le sujet étudié $[88,97]$.

Létude du comportement des juges peut constituer un thème de recherche en sociologie, mais la comparaison des jurys professionnels entre eux [12, $89,99,128-130,143,158]$ ou avec les jurys d'« hommes de la rue » $[3,12,42,75]$ a peu d'intérêt pour nous puisque le jury souverain est bien évidemment ce dernier.

La variabilité de l'attitude est cependant faible selon le sexe et l'âge; on note simplement une sévérité des juges masculins accrue pour les visages féminins $[51,52]$ et un œil très critique des adolescents pour tout défaut de la denture [50]. La morphologie faciale des juges a été envisagée comme un facteur de variation mais ceci n'a pas été confirmé [163].

\subsection{L'analyse de forme}

Lanalyse de la forme est le second pilier du jugement esthétique, dont le but est d'associer à chaque forme une cotation esthétique; le but est bien sûr thérapeutique : modeler la forme la plus belle possible.

Lanalyse va porter sur la coquille cutanée essentiellement, mais aussi sur la denture support des éléments cutanés esthétiquement les plus déterminants (lèvres, joues, commissures...) et jouant un rôle important lorsqu'elle est découverte (sourire), et sur la 
structure osseuse, support majeur de l'architecture cutanée.

Deux grandes directions s'offrent à nous, en 2D comme en 3D : la biométrie (céphalométrie) ou les méthodes comparatives issues de la morphométrie géométrique et basées principalement sur la superposition Procuste [121].

Ces méthodes diffèrent dans leur principe mais aussi :

- dans le mode de schématisation de la forme, qui conditionne d'ailleurs initialement l'emploi de l'une ou de l'autre,

- dans le choix de la zone de référence,

- et dans le mode de traitement statistique bien sûr ex post.

\subsubsection{La biométrie ou céphalométrie}

Elle va mesurer un certain nombre de paramètres géométriques : distances et vecteurs, angles et angles orientés (entre axes ou plans), surfaces gauches ou planes (surfaces polygonales définies par un diagramme de points), volumes.

Ces paramètres sont souvent constitués de ou liés aux coordonnées cartésiennes d'un diagramme de point schématisant la surface (coordonnées par rapport à un repère orthonormé choisi dans un zone stable et extérieure à la région d'analyse).

Lappréciation des dysmorphies reposera sur la comparaison de chaque mesure avec la valeur moyenne \pm l'écart-type. Un facteur correctif de taille peut être éventuellement introduit.

Les résultats (correction à effectuer ou effectuée pour «normaliser» la morphologie) seront immédiatement accessibles en millimètres et en direction.

Lapplication thérapeutique au diagnostic, au plan de traitement, au suivi de la croissance et/ou de l'action thérapeutique, est immédiate.

Ces méthodes «paramétriques » appellent une démarche passant par trois étapes :

- le choix des points-repères,

- le choix d'un ou plusieurs repères de référence,

- le choix des paramètres.

\subsubsection{Points-repères}

Le choix des points-repères est longuement débattu par divers auteurs [17-19,49, 54, 119-121].

$\mathrm{Au}$ niveau squelettique, architectural ou basimaxillaire, l'emploi des émergences trigéminales s'impose pour nous comme une évidence : elles ont le double avantage d'une détermination précise et non-opérateur dépendante, et d'un lien inducteur avec la morphogénèse maxillo-faciale. Nous avons donc adopté uniquement ces repères [54, 55, 120, 121] (Figs. 1 à 4).

Au niveau alvéolo-dentaire, les matrices d'inertie associées aux dents ou groupes de dents assurent un repérage optimal et automatisé.

La détermination des points repères cutanés pose un problème bien plus difficile. Il n'existe pas de points cutanés identifiables avec précision et sans erreur possible de l'opérateur; de plus, beaucoup de points intéressants sont en mouvement constant selon la déglutition, la mimique, etc. [91, 112]. Où sont les infra-orbitaires et les supra-orbitaires cutanés? Où est le point pro-nasal de la pointe du nez ?...

Aussi certains auteurs préfèrent-ils des méthodes mixtes : biométriques s'appuyant sur un repère basicrânien ou orbitaire pour l'alvéolo-dentaire et l'architectural, et avec des coquilles cutanées et des repérages colorés des différences morphologiques cutanées. Mais ceci pose le problème de la fusion.

\subsubsection{Repères de référence}

L'emploi d'un repère orthogonal de préférence lié à la base du crâne, comme celui que nous avons choisi, lié au pseudo-plan de Francfort, ou plan de Treil, permet d'envisager les dysmorphies dans un espace cartésien. L'emploi d'un référentiel lié à l'arcade permet d'employer le langage dentaire classique (direction verticale, mésio-distale, vestibulolinguale).

La compréhension de la dysmorphie est immédiate et distingue ce qui revient à chaque sens, alors que les échelles colorimétriques utilisées dans les logiciels de morphométrie géométrique caractérisent globalement l'écart morphologique document 1/document 2, souvent sans distinguer le sens.

\subsubsection{Paramètres}

Une céphalométrie complète doit disposer de points-repères au niveau alvéolo-dentaire (dents et arcades), au niveau basi-maxillaire (plan palatin et fer à cheval mandibulaire, au niveau architectural de l'ensemble cranio-facial [54, 120, 121]. Ainsi, elle pourra définir parfaitement une pathologie et 
envisager un plan de traitement précis, à partir de paramètres ici classés selon leur rôle fonctionnel :

- relation haut-bas (alvéolo-dentaire/basi-maxillaire/architecturale),

- compensation (relations basi-maxillaire/alvéolodentaire),

- dysmorphie commune haut et bas (biproalvéolie/birétroalvéolie),

- position d'une pièce anatomique/ repère,

- morphologie d'un élément anatomique (mandibule ou maxillaire).

Chacun d'eux produit des données dans les trois dimensions de l'espace et pour les zones antérieures, moyennes ou postérieures (selon la position de points repères).

La contrepartie de cette précision complète est l'excès de données, qui doit être géré par un logiciel d'intelligence artificielle. C'est ce que nous avons entrepris de faire.

Une fois résolus les trois problèmes que nous venons d'aborder, ces méthodes présentent l'avantage d'une précision totale distinguant les différents sens et les différents niveaux, et d'un traitement statistique simple et classique (test $t$, test $f$, matrice de corrélation, analyse en composantes principales (ACP) éventuellement).

Actuellement, aucune céphalométrie complète conduisant à une "aide au diagnostic » elle aussi complète, ne s'est imposée. Les publications récentes portent sur :

- Les points repères $[22,49,54,100,101,114,119$ 121, 149, 184] avec:

- une attention insuffisante pour l'aspect cutané (abordée en [22, 26, 83, 149]);

- une définition des repères squelettiques (avec une justification quelquefois arbitraire et sans un souci suffisant de leur fiabilité) [17, 18,32, 49, 54, 100, 101, 119-121, 184] ;

- un essai permanent de récupération des repères classiques du 2D, alors que c'est l'identification de points-repères définis directement dans une anatomie 3D qui devrait s'imposer.

- La comparaison des céphalométries 2D et 3D $[32,33,114,117]$, avec souvent encore une tentative de conservation des points-repères conventionnels du 2D, dans une reconstruction 3D à partir d'une saisie cone-beam [101] .
- La précision de la définition des points-repères identifiés en 3D [119, 183].

- Le set-up chirurgical trop souvent, négligeant le diagnostic et la définition de l'objectif orthodontique $[22,112,165]$.

\subsubsection{Les méthodes comparatives issues de la morphométrie géométrique et basées principalement sur la superposition Procuste}

Elles ont été d'abord introduites dans la recherche en anthropologie. Ce sont des méthodes de comparaison par superposition Procuste de deux formes identifiées par des diagrammes de pointsrepères anatomiques ou de pseudo-landmarks ou semi-landmarks obtenus par maillage.

La méthode est absolument opérateur-indépendante, et elle sépare sans erreur les différences de taille et les différences de forme.

Elle peut être employée en thérapeutique (diagnostic clinique, plan de traitement, suivi, recherche) en comparant par superposition le sujet étudié à la forme moyenne ou à un individu normo-morphique de référence. Toutefois, les outils sont plutôt des instruments de visualisation globale des différences que des outils de définition précise de la pathologie (direction et millimètres, niveau de l'atteinte: dentaire, basale, architecturale?).

\subsubsection{Méthodes issues de la morphométrie géométrique}

La morphométrie géométrique utilise classiquement les outils suivants [96] :

- superposition Procuste sur l'ensemble des pointsrepères,

- visualisation des différences par une échelle colorée,

- Analyse Procuste Généralisée (GPA Dryden et Mardia, 1993),

- analyse en couches minces (Thin Plate Analysis [19], Dryden et Mardia, 1993),

- test du caractère significatif des différences: Goodall test,

- analyse en composantes principales des différences (PCA Jolife 1986, Dunteman 1989), sélectionnant les sous-groupes,

- utilisation de la matrice des distances euclidiennes (EDMA : Euclidean Distance Matrix Analyses), 
- Wilk's Lambda : test de comparaison de forme, utilisé en analyse discriminante.

\subsubsection{Protocoles de comparaison utilisés en $O D F$}

Une meilleure mise en évidence des différences peut être obtenue par superposition sur une zone réduite considérée comme stable, c'est le « recalage ». Les références possibles sont la base du crâne (matérialisée par des points [55] ou la surface antérieure de la base du crâne [25,26], l'étage orbitaire [55]).

Selon Bookstein [17-19], l'emploi de points repères pour identifier la base du crâne serait insuffisant, c'est ce qui a conduit Cevidanes à utiliser une superposition sur des surfaces homologues des deux documents comparés [26].

Une superposition sur une zone stable met mieux en évidence les différences qu'une superposition sur l'ensemble bien sûr.

Cevidanes, et al. $[25,26]$ expliquent la démarche après saisie $\mathrm{CBCT}$ :

1. Construction des surfaces virtuelles 3D.

2. Enregistrement des images et superposition sur la région antérieure de la base du crâne.

3. Transparence.

4. Colorimétrie rendant compte des quantités de déplacement réalisé.

Certains auteurs superposent simplement les coquilles cutanées, soit en superposant au mieux les surfaces faciales délimitées, soit en utilisant des repères cutanés. Les points-repères utilisés sont :

- au nombre de 5 pour Kau et Richmond [91] : canthi internes des yeux, commissures, pointe du nez;

- au nombre de 13 pour Moerenhout, et al. [112] : sellion, alaires D et $G$, canthi internes D et $G$, pupillaires $D$ et $G$, sous-nasal, gnathion, zygions $D$ et $G$, supra-orbitaires D et $G$.

Ce type de superpositions donne une bonne représentation de l'évolution de la morphologie cutanée, mais ne la relie pas avec l'évolution squelettique et dentaire sous-jacente.

\subsubsection{Quelques résultats}

L'emploi de documents 3D comme supports de recherche en matière de morphologie ou d'esthétique faciale a déjà produit quelques résultats. Malheureusement, il s'agit surtout de résultats morphologiques, l'arbitrage souverain du juge n'ayant pas été envisagé.

- Méthode d'établissement des formes moyennes, ou stéréotypes [93, 178].

- Comparaison des standards ethniques $[93,95,180]$.

- Comparaison de sujets pathologiques avec des sujets normaux [76].

- Technologie des protocoles d'emploi du 3D : méthode d'imagerie cutanée: fiabilité des repères cutanés, fusion-intégration de coquilles cutanées avec les dents et l'architecture squelettique [25, 26, 175].

- Dynamique cutanée [105, 172, 178].

Mais ces méthodes apparemment opposées tendent à se rapprocher, vers une analyse mixte :

- céphalométrie pour l'architecture cranio-faciale et pour le diagnostic, le plan de traitement et le suivi du travail orthodontique envisagé,

- méthode d'appréciation esthétique à l'aide d'une saisie et d'une reconstitution 3D des tissus cutanés, soutenue par des superpositions:

- diagnostic (sur un sujet normo-morphique),

- simulation de traitement (superposition image de début/image modifiée simulant la thérapeutique envisagée par les données dentaires et osseuses),

- appréciation post-thérapeutique.

De gros progrès restent à faire :

- Au niveau céphalométrique, il reste à réaliser une biométrie complète, permettant un diagnostic complet avec, à partir des points repères choisis : l'analyse des paramètres déterminants, le diagnostic quantifié, à l'issue d'un logiciel d'intelligence artificielle, l'objectif de position dentaire et osseux défini et quantifié.

- Au niveau de l'esthétique cutanée, le choix d'un type de documents rendant compte de l'esthétique faciale en 3D reste en suspens.

- Au niveau des arcades dentaires, les modes de saisie 3D sont encore en discussion et la fusion fait l'objet aussi de plusieurs propositions.

\section{Conclusion}

À l'issue de ce panorama, il apparaît que nos connaissances sur l'abord social de l'esthétique faciale ont considérablement évolué au cours de ces 
vingt dernières années, mais d'une manière assez inégale.

Les méthodes, tant au niveau des supports qu'au niveau du traitement statistique, sont aujourd'hui bouleversées par l'apparition du 3D : les images tridimensionnelles présentent un rendu extrêmement vivant, les moyens de comparaison, issus en grande partie des protocoles de la morphométrie géométrique, autorisent des analyses sans biais.

Déjà, à l'horizon, on voit pointer les images dynamiques, encore un grand pas en avant!

Les connaissances fondamentales n'ont pas, pour nous, progressé à l'aune des avancées techniques précédemment décrites : au niveau des facteurs morphologiques de la préférence esthétique, c'est la littérature orthodontique ou chirurgico-orthodontique qui joue le rôle moteur. Pour tous les autres facteurs, c'est dans les revues scientifiques de sociologie qu'on trouve les principales avancées. Les recherches semblent freinées par l'extrême variabilité dans l'espace et dans le temps des préférences ou des critères esthétiques, qui sont liés, nous l'avons vu, à la culture du groupe social environnant.

Or le thérapeute comme le chercheur se doit de surfer sur la vague de la mode, d'en saisir le profond ou le durable et d'en éliminer le futile ou le superflu. Il faut en effet fabriquer l'esthétique faciale qui plaît à notre patient, et même celle qui lui plaira encore dans dix ans, sans bien sûr négliger les exigences d'équilibre musculaire, de stabilité, etc. Il faut donc parfaitement maîtriser l'esthétique d'aujourd'hui et même anticiper l'esthétique de demain.

Sans entrer trop dans le détail de ces évolutions de l'appréciation esthétique, relisons une fois encore les superbes pages de «L'homme révolté » où Camus, ce grand humaniste, nous explique les évolutions cycliques de l'art, dans l'œil de l'amateur comme dans le génie du créateur, en fonction des révoltes et des périodes révolutionnaires succédant aux périodes conservatrices ou de contre-réformes.

\section{Bibliographie}

[1] Ackerman M, Ackerman J. Smile paralysis and design in the digital era. J Clin Orthod 2002;36:221-236.

[2] Ackermann MB. Digital video as a clinical tool in orthodontics: dynamic smile design in diagnosis and treatment planning. In: Mc Namara JA, Ed. 29 ${ }^{\text {th }}$ Annual Moyer's symposium. Vol 40. Ann Arbor: University of Michigan, Department of Orthodontics, 2003.
[3] Albino JE, Tedesco LA, Conny DJ. Patient perceptions of dental facial esthetics : shared concerns in orthodontics and prosthodontics. J Prosth Dent 1984;52:9-13.

[4] Albino JE, Tedesco LA, Kiyak HA. Esthetic issues in behavioral dentistry. Ann Behav Med 1990;12:148-155.

[5] Albino JE, Tedesco LA. Esthetic need for orthodontic treatment. In : Melsen B. Ed. Current controversies in orthodontics. Chicago : Quintessence Publishing Co. Inc., 1991:11-24.

[6] Albino JE. Development of methodology for behavioral measurements related to malocclusion (Final Report: Contract $N^{\circ}$, NO1-DE-27499). Bethesda, MD: National Institute of Dental Research, 1981.

[7] Al-Khateeb SH. Perception of facial profile attractiveness of different antero-posterior and vertical proportion. Eur J Orthod 2010;32 (4).

[8] Alley TR, Cunningham MR. Averaged faces are attractive but very attractive faces are not average. Psychol Sci 1991;2:123-125.

[9] Arnett GW, Bergman RT. Facial keys to orthodontic diagnosis and treatment planning. Part I. Am J Orthod Dentofac Orthop;03:299-312.

[10] Arnett GW, Bergman RT. Facial keys to orthodontic diagnosis and treatment planning. Part 2. Am J Orthod Dentofac Orthop 1993;103:395-441.

[11] Bayome M, Park JH, Kook YA. New three dimensional cephalometric analyses among adults with a skeletal Class I pattern and normal occlusion. Korean J Orthod 2013;43:62-73.

[12] Bell R, Kiyak HA, Joondeph DR, McNeill RW, Wallen TR. Perception of facial profile and their influence on the decision to undergo orthognathic surgery. Am J Orthod 1985;88:323-332

[13] Benson P, Perrett DI. Face to face with the perfect image. New Scientist 1992;133:32-35.

[14] Bertamini M, Bennet KM. The effect of leg length on perceived attractiveness of simplified stimuli. J Soc Evol Cult Psychol 2009;3:233-250.

[15] Bishara SE, Jakobsen JR. Profile changes in patients treated with and without extractions : Assessment by lay people. Am J Orthod Dentofac Orthop 1997;112:639-644.

[16] Bittner C, Pancherz H. Malocclusions et morphologie faciale. Am J Orthod Dentofac Orthop (Ed Fr) 1990;2:270-278.

[17] Bookstein FL. Landmark methods for forms without landmarks: localizing group differences in outline shape. Med Image Anal 1997;1:5-16.

[18] Bookstein FL. Applying landmark methods to biological outline data. In: Mardia KV, Gill CA, Dryden II editors. Image fusion and shape variability. Leeds UK: University of Leeds Press 1966:59-70.

[19] Bookstein FL. Size and shape spaces for landmark data in two dimension. Statistical Science 1986;1:181-242.

[20] Burstone CJ. Integumental contour and extension patterns. Angle Orthod 1959;29:93-104.

[21] Buss D. The evolution of desire ( ${ }^{\text {nd }}$ ed.). New York: Basic Books, 2003:54-55. 
[22] Calignano F, Vezzetti E. Soft tissue diagnosis in maxillofacial surgery: a preliminary study on three-dimensional face geometrical features-based analysis. Aesthetic Plast Surg 2010;34:200-211.

[23] Camara CA. Aesthetics in Orthodontics : Six horizontal smile lines. Dental Press J Orthod 2010;15:116-131.

[24] Caruso AJ, Stanhope SI, McGuire DA. New technique for acquiring three-dimensionnal orofacial nonspeech motion. Dysphagia 1989;4:127-132.

[25] Cevidanes LHC, Figueiredo Oliveira AE, Grauer D, Styner M, Proffit WR. Clinical Application of 3D Imaging for Assessment of Treatment Outcomes. Semin Orthodontics 2011;17:72-80.

[26] Cevidanes LHC, Motta A, Proffit WR, Ackerman JL, Stynere M. Cranial base superimposition for 3-dimensional evaluation of soft-tissue changes. Am J Orthod Dentofac Orthop 2010;137:120-129.

[27] Cochrane SM, Cunningham SJ, Hunt NP. A comparison of the perception of facial profile by the general public and three groups of clinicians. Int J Adult Orthod Orthognath Surg 1999;14:291-295.

[28] Cunningham MR. Measuring the physical in physical attractiveness/ Quasi-experiments on the sociobiology of Female Beauty. J Pers Soc Psychol 1986,50:925-935.

[29] Cunningham SJ. The psychology of facial appearance. Dent Update 1999;26:438-443.

[30] Cunningham MR, Roberts AR, Barbee AP, Druen PB, Wu $\mathrm{CH}$. Their ideas of beauty are, on the whole, the same as ours: Consistency and variability in the cross-cultural perception of female physical attractiveness. J Pers Soc Psychol 1986;68:261-279.

[31] Czarnecki ST, Nanda RS, Currier GF. Perceptions of a balanced facial profile. Am J Orthod Dentofac Orthop 1993,104:180-187.

[32] Damstra J, Fourie Z, De Witt M, Ren Y. A three dimensional comparison of a morphometric and conventional cephalometric midsagittal plan for craniofacial asymmetry. Clin Oral Investig 2012;16:285-294.

[33] Damstra J, Fourie Z, Ren Y. Comparison between twodimensional and midsagittal three-dimensional cephalometric measurements of dry human skulls. Br J Oral Maxillofac Surg 2011;49:392-395.

[34] Davis ST, Jahnke JC. Unity and the golden section : rules for aesthetic choice? Am J Psychol 1991;104:257-277.

[35] DeBruine LM, Jones BC, Crawford JR, Welling LL, Little AC. The health of a nation predicts their mate preferences: cross-cultural variation in women's preferences for masculinized male faces. Proc R Soc B London 2010;277:2405-2410.

[36] De Coster Th. Etude comparative des critères d'évaluation céphalométrique du profil cutané. Orthod Fr 1991;62:559-572.

[37] De Vos W, Casselman J, Swennen GR. Cone-beam computerized tomography (CBCT) imaging of the oral and maxillofacial region: A systematic review of the literature. Int J Oral Maxillofac Surg 2009;38:609-625.

[38] Deffenbacher KA, Vetter T, Johanson J, O'Toole AJ. Facial aging, attractiveness, and distinctiveness. Perception 1998;27:1233-1243.
[39] Dixson BJ, Dixson AF, Bishop PJ, Parish A. Human physique and sexual attractiveness in men and women: a New Zealand-US Comparative Study. Arch Sex Behav 2010;99:798-806.

[40] Dixson BJ, Dixson AF, Li B, Anderson MJ. Studies of human physique and sexual attractiveness: sexual preferences of men and women in China. Am J Hum Biol 2007;19:88-95.

[41] Dixson BJ, Halliwell G, East R, Whignarajah P, Anderson MJ. Masculine Somatotype and hirsuteness as determinants of sexual attractiveness to women. Arh Sex Behav 2003:32:29-39.

[42] Dunlevy HA, White RP, Turvey TA. Professional and lay judgment of facial esthetic changes following orthognathic surgery. Int J Adult Orthod Orthognath Surg 1987;3:151-158.

[43] Eagly AH, Ashmore RD, Makhijani MG, Longo LC. What is beautiful is good, but...: a meta-analytic review of research on the physical attractiveness stereotype. Psychol Bull 1991;110:109-128.

[44] Edler RJ. Background Considerations to Facial Aesthetics. J Orthod 2001;28:159-168.

[45] Ekman P, Davidson RJ, Friesen WV. The Duchenne Smile/Emotion expression and brain physiology II. J Pers Soc Psychol 1990;58;342-353.

[46] Ekman P. Hand Book of Cognition and Emotion 1990 T. Dalgleish and Power. Sussex UK. John Wiley \& sons Ltd.

[47] English BG, Ashmore R. Beauty before the eyes of the beholder : the cultural encoding of beauty types in magazine advertising and music television. J Advertising 1994,23:49-64.

[48] Erbay EF, Caniklioğlu CM. Soft tissue profile in Anatolian Turkish adults: Part II. Comparison of different soft tissue analyses in the evaluation of beauty. Am J Orthod Dentofac Orthop 2002;121:65-72.

[49] Farronato G, Garagiola U, Dominici A, Periti G, de Nardi S, Carletti V, Farronato D. "Ten-point" 3D cephalometric analysis using low-dosage cone beam computed tomography. Prog Orthod 2010;11:2-12.

[50] Faure J, Baron P, Justumus P. Agénésies des incisives latérales supérieures: évaluation statistique des traitements par substitution. Rev Orthop Dento Faciale 1994;28:211-224

[51] Faure J, Casteigt J. Chirurgie orthognathique: le préjudice esthétique initial et son amélioration thérapeutique. Rev Orthop Dento Faciale 1997,31:319-327.

[52] Faure J, Cisse A, N'Dindin-Guigan E, Aka A, Roux H, Diagne F. Appréciation du préjudice esthétique lié aux dysmorphoses maxillo-faciales en Afrique de l'Ouest. Rev Odonto-Stomatol-Chir Maxillo-Fac Afr 1997;4:30-40.

[53] Faure J, Guignes P. Appreciation of therapeutic results: an esthetic test. 31st Annual Meeting Lyon, 16-17 september 1994 [Abstract]. J Dent Res 1995;74:196.

[54] Faure J, Marshal-Sixou Ch, Dah-Jouonzo H, Oueiss A, Baron P. Three dimensionnal 14 points cephalometric analysis: compensation of maxillo-facial dysmorphisms. Orthod Fr 2008;79:251-261

[55] Faure J, Oueiss A, Treil J, Braga J. Etude en morphométrie géométrique des asymétries faciales: rapports 
avec les formes basi-crâniennes et les pathologies maxillo-faciales ou occlusales. Rev Orthop Dento Faciale 2013;47:157-171.

[56] Faure J. Le préjudice esthétique des grandes dysmorphies antéro-postérieures et verticales : son évolution après traitement orthodontique. Rev Orthop Dento Faciale 1998;32:275-295

[57] Faure J. Limite chirurgicale en classe II hyperdivergente. J Edg 1994;29:47-57.

[58] Faure J. A propos des troubles morphologiques du groupe incisivo-canin supérieur, chez deux sœurs jumelles. Rev Orthop Dento Faciale 2012; 46:427-441.

[59] Faure J. Gestion quantitative des troubles morphologiques du groupe incisive-canin. Rev Orthop Dento Faciale 2012;46:417-425.

[60] Frederik DA, Fessler DMT, Haselton MG. Do representation of male muscularity differ in men's and women's magazines? Body Image 2004;V2:81-86.

[61] Freedman FE, Carter MM, Sbrocco T, Gray JJ. Do men hold African-American and Caucasian women to different standards of beauty. Eat Behav 2007;8:319-333.

[62] Freedman FE, Carter MM, Sbrocco T, Gray JJ. Ethnic differences in preference for female weight and waist-tohip ratio: a comparison of African-American and white American college and community samples. Eat Behav 2004;5:191-198.

[63] Frindel F. Pour un meilleur positionnement du sourire. Rev Orthop Dento Faciale 2001;35:473-497; 2002;36:82-92.

[64] Furnham A, Tan T, Mc Manus C. Waist-to-hip ratios and preferences for body shape: A replication and extension. Pers Individ Dif 1997;22:539-549.

[65] Giddon DB, Sconzo R, Kinchen JA, Evans CA. Quantitative comparison of computerized discrete and animated profile preference. Angle Orthod 1995;66:441-448.

[66] Giddon DB, Bernier DL, Kinchen JA, et al. Comparison of computer-animated programs for quantifying facial profile preference [Abstract]. J Dent Res 1995;74:140.

[67] Giddon DB, Hershon LE, Lennartson B. Discrepancy between objective and subjective profile measures. Scand J Dent Res 1974,82:527-535.

[68] Giddon DB, Sconzo R, Kinchen JA, Evans CA. Psychological comparison of computerized discrete and animated profile preferences [Abstract]. J Dent Res 1994;73:21-29

[69] Giddon DB. Orthodontic applications of psychological and perceptual studies of facial esthetics. Seminars Orthod 1995;1:82-93.

[70] Glassenberg AN, Feinberg DR, Jones BC, Little AC, Debrunine LM. Sex-dimorphic face shape preference in heterosexual and homosexulanmen and women. Arch Sex Behav 2010;39:1286-1296.

[71] Goshman DS. Some correlates of children's health beliefs and potential health behaviour. J Health Soc Behav 1972,12:148-154.

[72] Goshman DS. The measurement and development of dentally relevant motives. J Health Soc Behav 1975;35:160-164.
[73] Gross MM, Trotman CA, Moffatt KS. A comparison of threedimensional and twodimensionnal analyses of facial motion. Angle Orthod 1996;66:189-194.

[74] Halberstadt J, Rhodes G. It's not just average faces that are attractive: computer-manipulated averageness makes birds, fish, and automobiles attractive. Psychon Bull Rev 2003;10:149-156.

[75] Hall D, Taylor RW, Jacobson A, Sadowsky PL, Bartolucci A. The perception of optimal profile in African Americans versus white American as assessed by orthodontists and the lay public. Am J Orthod Dentofac Orthop 2000;118:514-525.

[76] Hanis SB, Kau CH, Souccar MM, English JD, Pirttiniemi P, Valkama M, Harila V. Facial morphology of Finnish children with and without developmental hip dysplasia using 3D facial templates. Orthod Craniofac Res 2010;13:229-237.

[77] Haxton JM, Giddon DB. Quantitation of tolerance for cranio-facial deviation [Abstract]. J Dent Res 1985,64:292.

[78] Hershon LE, Giddon DB. Determinants of facial profile self-perception. Am J Orthod 1980;78:279-295.

[79] Hier LA, Evans CA, BeGole EA, Diddon DB. Comparison of preferences in lip position using computer animated imaging. Angle Orthod 1999;69:231-238.

[80] Hoss K, Sameshima GT, Grubb JE, Sinclair PM. The accuracy of video imaging for mixed dentition and adolescent treatment. Angle Orthod 1997;67:355-364.

[81] Hunt O, Hepper P, Johnston C, Stevenson M, Burden D. The aesthetic component of the index of orthodontic treatment validated against lay opinion. Eur J Orthod 2002;24:53-59.

[82] Hunt O, Johnston C, Hepper P, Burden D, Stevenson M. The influence of maxillary gingival exposure on dental attractivenes ratings. Eur J Orthod;24:199-204.

[83] Incraperaa AK, Kau CH, English JD, McGroryd K, Sarvere DM. Soft Tissue Images from Cephalograms Compared With Those from a3D Surface Acquisition System. Angle Orthod 2010;80:58-64.

[84] Jefferson Y. Facial Beauty: Establishing a Universal Standard. IJO;15:9-22.

[85] Jenny J, Cons NC, Kohout F, Frazier PJ. Test of a method to determine socially acceptable occlusal conditions. Los Angeles, CA: American Association for Dental Research 1980.

[86] Johnson DK, Smith RJ. Smile esthetics after orthodontic treatment with and without extraction of four first premolars. Am J Orthod Dentofac Orthop 1995;108:162-167.

[87] Johnston CD, Burden DJ, Stevenson MR. The influence of dental to facial midline discrepancies on dental attractiveness ratings. EJO 1999;21:517-522.

[88] Johnston V, Franklin M. Is beauty in the eye of the beholder? Ethol Sociobiol 1993;14:183-199.

[89] Jones D, Hill K. Criteria of facial attractiveness of five populations. Hum Nature 1993;4:271-296.

[90] Kanazawa S. Intelligence and physical attractiveness. Intelligence 2011;39:7-14. 
[91] Kau CH, Richmond S. Three-dimensional analysis of facial morphology surface changes in untreated children from 12 to 14 years of age. Am J Orthod Dentofac Orthop 2008;134:751-760.

[92] Kau CH, Richmond S, Incrapera AK, English J, Xia JJ. Three-dimensional surface acquisition systems for the study of facial morphology and their application to maxillofacial surgery. Int J Med Robotics Comput Assist Surg 2007;3:97-110.

[93] Kau CH. Creation of the virtual patient for the study of facial morphology. Facial Plast Surg Clin North Am 2011;19:615-22, viii.

[94] Kau CH, Zhurov A, Richmond S, Bibb R, Sugar A, Knox J, Hartles F. The 3-Dimensional Construction of the Average 11-Year-Old Child Face: A Clinical Evaluation and Application. J Oral Maxillofac Surg 2006;64:1086-1092.

[95] Kau, CH, Richmond S, Zhurov A, Ovsenik M, Tawfik W, Borbely P, Englishg JD. Use of 3-dimensional surface acquisition to study facial morphology in 5 populations. Am J Orthod Dentofac Orthop 2010;137:S56.e9.

[96] Kendall G. Shape manifolds, Procustean metrics and complex projective spaces. Bull London Math Soc 1984;16:81-121.

[97] Kissler J, Bäuml KH. Effects of the beholder's age on the perception of facial attractiveness. Acta Psychol 2000;104:145-166.

[98] Kiyak HA, Zeitler DL. Self-assessment of profile and body image among orthognathic surgery patients before and two years after surgery. J Maxillofac Surg 1988;46:365-371.

[99] Kokich VO, Kiyak HA, Shapiro PA. Comparing the perception of dentists and lay people to altered dental esthetics. J Esthet Dent 1999;11:311-324.

[100] Lagravere MO, Gordon JM, Guedes IH, Flores-Mir C, Carey JP, Heo G, Major PW. Reliability of traditional cephalometric landmarks as seen in three-dimensional analysis in maxillary expansion treatments. Angle Orthod 2009;79:1047-1056.

[101] Lagravere MO, Mayor PW, Carey J. Sensitivity analysis for plane orientation in three dimensional cephalometric analysis based on superimposition of serial cone beam computed tomography images. Dentomaxillofacial Radiol 2010;39:400-408.

[102] Langlois JH, Kalanikis, Rubenstein AJ, Larson A, Hallam M, Smoot M. Maxims or Myths of Beauty? A meta-analytical and theoretical review. Psychol Bull 2000;126:390-423.

[103] Langlois JH, Roggman LA, Musselman L. What is average and what is not average about attractive faces? Psychol Sci 1994;5:214-220.

[104] Langlois JH, Roggman LA. Attractive faces are only average. Psychol Sci 1990;1:115-121.

[105] Lee JY, Han Q, Trotman CA. Three-dimensional facial imaging: accuracy and considerations for clinical applications in orthodontics. Angle Orthod 2004;74:587-593.

[106] Lim JY, Giddon DB. Self/other perception and facial anthropometry in Korean (K), Korean-American (KA) and Caucasian (C) females [Abstract]. J Dent Res 1991;70:430.
[107] Little AC, Burt DM, Penton-Voak IS, Perrett DI. Selfperceived attractiveness influences human female preferences for sexual dimorphism ans symmetry in male faces. Proc R Soc London B Biol Sci 2001;268:39-44.

[108] Maxwell R, Kiyak HA. Dentofacial appearance : a comparison of patient self-assessment techniques. Int J Adult Orthod Orthognath Surg 1991;6:123-131.

[109] McKoy-White J, Evans CA, Viana G, Anderson NK, Giddone DG. Facial profile preferences of black women, before and after orthodontic treatment. Am J Orthod Dentofac Orthop 2006;129:17-23.

[110] Mealey L, Bridgstock R, Townsend GC. Symmetry and perceived facial attractiveness: a monozygotic co-twin comparison. J Pers Soc Psychol 1999;76:151-158.

[111] Michiels G, Sather AH. Determinants of facial attractiveness in a sample of white women. Int J Adult Orthod Orthognath Surg 1994;9:95-103.

[112] Moerenhout BAMML, Gelaude F, Swennen GRJ, Casselman JW, Van der sloten J, Mommaerts JMY. Accuracy and repeatability of cone-beam computed tomography (CBCT) measurements used in the determination of facial indices in the laboratory setup. J Cranio-Maxillofac Surg 2009;37:18-23.

[113] Morley J. The role of cosmetic dentistry in restauring of youthfull appearance. 1999. J Am Dent Assoc 130:1166-1172.

[114] Moro A, Correra P, Boniello R, Gasparini G, Pelo S. Threedimensional analysis in facial asymmetry: comparison with model analysis and conventional two-dimensional analysis. J Craniofac Surg 2009;20:417-422.

[115] Myers PN, Biocca FA. The elastic body image: the effect of television advertising and programming on body image distorsions in young women. J Commun 1992;42:108-133.

[116] Nakajima E, Maeda T, Yanagisawa M. The Japanese sense of beauty and facial proportions II. The beautiful face and the $\sqrt{2}$ rule. Quintessence Internat 1985;9:629-637.

[117] Nalçaci R, Öztürk, F, Sökücü O. A comparison of twodimensional radiography and three-dimensional computed tomography in angular cephalometric measurements. Dentomaxillofac Radiol 2010;39:100-106.

[118] Nguyen DD, Turley PK. Changes in the caucasian male facial profile as depicted in fashion magazines during the twentieth century. Am J Orthod Dentofac Orthop 1998;114:208-217.

[119] Olszewski R, Tanesy O, Cosnard G, Zech F, Reychler H. Reproducibility of osseous landmarks used for computed tomography based three-dimensional cephalometric analyses. J Craniomaxillofac Surg 2010;38:214-221.

[120] Oueiss A, Pages C, Treil J, Braga J, Baron P, Faure J. Étude des asymétries dans les grandes dysmorphies antéropostérieures. Orthod Fr 2010;81:235-244.

[121] Oueiss A. Les rapports tridimensionnels de la base du crâne et du massif maxillo-facial : intérêt en orthodontie et en anthropobiologie. Thèse Doctorat d'Université Toulouse : Univ Toulouse III, 2010.

[122] Peck H, Peck S. A concept of facial esthetics. Angle Orthod 1970;40:284-318. 
[123] Peck S, Peck L. Selected aspects of the art and science of facial esthetics. Semin Orthod 1995;1:105-126.

[124] Peck S. Beauty is youth, youth beauty? Sci News 1994;146:115

[125] Penton-Voak IS, Jones BC, Little AC, Baker S, Tiddeman B, Burt DM, Perrett DI. Symmetry, sexual dimorphism in facial proportions and male facial attractiveness. Proc R Soc London B Biol Sci 2001;268:1617-1623.

[126] Perrett DI, May KA, Yoshikawa S. Facial shape and judgements of female attractiveness. Nature 1994,368:239-242.

[127] Perrett DI, Lee KJ, Penton-Voak IS, Rowland DR, Yoshikawa S, Burt DM, Henzi SP, Castles DL, et al. Effect of sexual dimorphism on facial attractiveness. Nature 1998;394:884-887

[128] Phillips C, Griffin T, Bennett E. Perception of facial attractiveness by patients, peers, and professionals. Int J Adult Orthod Orthognath Surg 1995;10:127-135.

[129] Pietila T, Pietila I. Parent's views on their own child's dentition compared with an orthodontist's assessment. Eur J Orthod 1994;16:309-316.

[130] Prahl-Andersen B, Boersma H, Van der Linden FPGM, Moore AW. Perceptions of dentofacial morphology by laypersons, general dentists, and orthodontists. J Am Dent Assoc 1979,98:209-212.

[131] Prahl-Anderson B. The need for orthodontic treatment. Angle Orthod 1978;48:1-9.

[132] Principe CP, Langlois JH. Shifting the prototype. Experience with faces influences affective and affectiveness preference. Soc Cogn 2006;30:109-120.

[133] Rankin M, Borah GL. Perceived functional impact of abnormal facial appearance. Plast Reconst Surg 2003;111:2140-2146.

[134] Ramsey-Rennels JL, Langlois JH. Infants' differential Processing of Female and Male Faces. Psychol Sci 2005; 15:50-62.

[135] Rhodes G, Yoshikawa S, Clark A, Lee K, McKay R, Akamatsu S. Attractiveness of facial averageness and symmetry in non-Western cultures : in search of biologically based standards of beauty. Perception 2002;30:611-625.

[136] Richins ML. Social comparison and the idealized images of advertising. J Consumer Research 1991;18:71-83.

[137] Ricketts RM. Divine proportions in facial esthetics. Clin Plast Surg 1982;9:401-422.

[138] Ricketts RM. The biological significance of the divine proportion and fibonacci series. Am J Orthod 1982;81:351-370.

[139] Rikowski A, Grammer K. Human body odour, symmetry and attractiveness. Proc Biol Sci 1999;266:869-874.

[140] Ritter DE, Gandini LG, dos Santos Pinto A, Ravelli DB, Lock A. Analysis of the smile photograph. Word J Orthod 2006; 7:279-285.

[141] Ritter JM, Casey RJ, Langlois JH. Adults' response to infants varying in apperance of age and attractiveness. Child Dev 1991;62:68-82.

[142] Robins AH. Biological perspectives on human pigmentation. Cambridge University Press 1, 1991.
[143] Romani KL, Agahi F, Nanada R, Zernik JH. Evaluation of horizontal and vertical differences in facial profiles by orthodontists and lay people. Angle Orthod 1993;63:175-182.

[144] Rubenstein AJ, Kalakanis L, Langlois JH. Infant preferences for attractive faces: a cognitive explanation. Dev Psychol 1999;35:848-855.

[145] Sarver DM, Ackerman MB. Dynamic smile visualization and quantification: Part 1. Evolution of the concept and dynamic records for smile capture. Am J Orthod Dentofac Orthop 2003;124:4-12.

[146] Sarver DM. The importance of incisor positioning in the esthetic smile: The smile arc. Am J Orthod Dentofac Orthop 2001;120:98-111

[147] Seagera DC, Kau CH, English JD, Tawfikd W, Bussae HI, El Yazeed A, Ahmedf M. Facial morphologies of an adult Egyptian population and an adult Houstonian white population compared using 3D imaging. Angle Orthod 2009;79:991-999.

[148] Secord PF, Backman CW. Malocclusion and psychological factors. J Am Dent Assoc 1959;59:931-938.

[149] Sforza C, de Menezes M, Ferrario VF. Soft- and hardtissue facial anthropometry in three dimensions: what's new. J Anthropol Sci 2013;91:1-26.

[150] Shaw WC, Meek SC, Jones DS. Nicknames, teasing, harassment and the salience of dental features among school children. Br J Orthod 1980;7:75-80.

[151] Shaw WC. Influence of children's dental-facial appearance on their social attractiveness as juged by peers and lay adults. Am J Orthod 1981;79:399-415.

[152] Shelly AD, Southard TE, Southard KA, Casko JS, Jakobsen JR, Fridrich KL, Mergen JL. Evaluation of profile esthetic change with mandibular advancement surgery. Am J Orthod Dentofac Orthop 2000;117:630-637.

[153] Singh D. Female judgment of male attractiveness and desirability for relationships: Role of waist-tohip ratio and financial status. J Pers Soc Psychol 1995;69(6):1089-1101

[154] Snow S.R. Esthetic smile analysis of maxillary anterior tooth width: The golden percentage. J Esthet Dent 1999;11:177-184.

[155] Sorokowski P, Pavlovski B. Adaptive preference for leg length in a potential Partner. Evol Hum Behav 2008;29:86-91.

[156] Sorokowski P. Attractivenes of legs length in Poland and Great Britain. J Hum Ecol 2000;31:148.

[157] Spyropoulos MN, Halazonetis DJ. Significance of soft tissue profile on facial esthetics. Am J Orthod Dentofac Orthop 2001;119:461-471.

[158] Stenvik A, Espeland L, Linge BO, Linge L. Lay attitudes to dental appearance and need for orthodontic treatment. Eur J Orthod 1997;19:271-277.

[159] Stephen I, Coetzee V, Law Smith MJ. Carotenoid and melanine pigment affect perceived human health. Evol Hum Behav 2011:32:216-227.

[160] Stephen I, Coetzee V, Law Smith MJ, Perrett DI. Skin blood perfusion and oxygenation affects perceived human health. PLoS One 2009. 4:e 5083 
[161] Stephen I, Law Smith MJ, Stirrat MR, Perrett DI. Facial skin coloration affects perceived health of human faces. Int J Primatol 2009;30:845-857.

[162] Stromboni Y. Facial aesthetics in Orthodontic Treatments with and without extractions. Eur J Orthod 1979;1:201-206

[163] Strzalko J, Kaszycka KA. Physical attractiveness: interpersonal and intrapersonal variability of assessments. Soc Biol 1992;39:170-176.

[164] Swennen GRJ, Mommaerts MY, Abeloos J, De Clercq C, Lamoral P, Neyt N, Casselman J, Schutyser F. A conebeam $\mathrm{CT}$ based technique to augment the 3D virtual skull model with a detailed dental surface. Int J Oral Maxillofac Surg 2009;38:48-57.

[165] Swennen GRJ, Mollemans W, Schutyser F. ThreeDimensional Treatment Planning of Orthognathic Surgery in the Era of Virtual Imaging. J Oral Maxillofac Surg 2009;67:2080-2092.

[166] Tatarunaite E, Playle R, Hood K, Shaw W, Richmond S. Facial attractiveness: A longitudinal study. Am J Orthod Dentofacial Orthop 2005;127:676-682.

[167] Tedesco LA, Albino JE, Cunat JJ, Slakter MJ, Waltz KJ. A dental-facial attractiveness scale: Part II, consistency of perception. Am J Orthod 1983,83:44-46.

[168] Thornhill R, Gangestad SW. Facial attractiveness. Trends Cogn Sci 1999;3:452-460.

[169] Tjan AHL, Miller GD. The JPG. Some esthetic factors in a smile. J Prosthetic Dent 1984;51:24-28 .

[170] Trotman CA, Gross MM, Moffatt KS. Reliability of a three dimensional method for measuring facial animation: a case report. Angle Orthod 1996;66:195-198.

[171] Trotman CA, Faraway JJ. Modeling facial movement: I A dynamic analysis of differences based on skeletal characteristics. J Oral Maxillofac Surg 2004;62:1372-1379.

[172] Trotman CA, Faraway J, Soltmann R, Hartman T, van Aalst J. Facial soft tissue dynamics before and after primary lip repair. Cleft Palate Craniofac J 2013;50:315-322.

[173] Trotman CA. Faces in 4 dimensions: Why do we care, and why the fourth dimension? Am J Orthod Dentofac Orthop 2011;140:895-899.

[174] Tucker S, Cevidanes LHS, Styner M, Kim H, Reyes M, Proffit W, et al. Comparison of actual surgical outcomes and 3Dimensional surgical simulations. J Oral Maxillofac Surg 2010;68:2412-2421.

[175] Uechi J, Okayama M, Shibata T, Muguruma T, Hayashi K, Endo K, Mizoguchi I. A novel method for the 3-dimensional simulation of orthognathic surgery by using a multimodal image-fusion technique. Am J Orthod Dentofac Orthop 2006;130:786-798.

[176] Vargo JK, Gladwin M, Ngan P. Association between ratings of facial attractivess and patients' motivation for orthognathic surgery. Orthod Craniofac Res 2003;6:63-71.

[177] Voracek M, Fisher ML, Rupp B, Lucas D, Fessler DM. Sex differences in relative foot length and perceived attractiveness of female feet: relationship among anthropometry, physique and preference rating. Percept Mot Skills 2007;104:1123-1138.

[178] Weeden JC, Trotman CA, Faraway JJ. Three dimensional analysis of facial movement in normal adults: influence of sex and facial shape. Angle Orthod 2001;71:132-140. Erratum in: Angle Orthod 2001;71:525.

[179] Werli T, Mathis R, Hédelin G, Rothea-Gouillard C. Evaluation esthétique du profil cutané par des étudiants en arts plastiques. Rev Orthop Dento Faciale 2003;37:145-157.

[180] Wirthlin J, Kau CH, English JD, Pan F, Zhou H. Comparison of facial morphologies between adult Chinese and Houstonian Caucasian populations using three-dimensional imaging. Int J Oral Maxillofac Surg 2013;42:1100-1107.

[181] Xu TM, Kom EL, Liu Y, Oh HS, Lee KH, Boyd RL, Baumrind S. Facial Attractiveness: Ranking of end of treatment facial photographs by pairs of Chinese and US Orthodontists. Am J Orthod Dent Orthop 2008;134:74-84.

[182] Young JA, Critelli JW, Keith KW. Male age preferences for short term and long term mating. Sex Evol Gender 2005; 7:83-93.

[183] Zachrisson BU. Esthetic factors involved in anterior tooth display and the smile: vertical dimension. J Clin Orthod 1998;32:432-445.

[184] Zamora N, Llamas JM, Cibrin R, Gandia JL, Oaredes V. A study on the reproductibility of cephalometric landmarks when undertaking a three-dimensional (3D) caphalometric analysis. Med Oral Pathol Oral Cir Buccal 2012;17:678-688. 